\title{
A class of three-dimensional gyroviscous magnetohydrodynamic models
}

\author{
Manasvi Lingam ${ }^{\circledR 1,2, \dagger}{ }^{1}$, Philip J. Morrison ${ }^{\circledR 3}$ and Alexander Wurm ${ }^{4}$ \\ ${ }^{1}$ Department of Aerospace, Physics and Space Sciences, Florida Institute of Technology, \\ Melbourne, FL 32901, USA \\ ${ }^{2}$ Institute for Theory and Computation, Harvard University, Cambridge, MA 02138, USA \\ ${ }^{3}$ Department of Physics and Institute for Fusion Studies, The University of Texas at Austin, \\ Austin, TX 78712, USA \\ ${ }^{4}$ Department of Physical and Biological Sciences, Western New England University, \\ Springfield, MA 01119, USA
}

(Received 27 February 2020; revised 5 August 2020; accepted 6 August 2020)

\begin{abstract}
A Hamiltonian and action principle formalism for deriving three-dimensional gyroviscous magnetohydrodynamic models is presented. The uniqueness of the approach in constructing the gyroviscous tensor from first principles and its ability to explain the origin of the gyromap and the gyroviscous terms are highlighted. The procedure allows for the specification of free functions, which can be used to generate a wide range of gyroviscous models. Through the process of reduction, the noncanonical Hamiltonian bracket is obtained and briefly analysed.
\end{abstract}

Key words: plasma dynamics, plasma nonlinear phenomena, plasma properties

\section{Introduction}

The importance of finite Larmor radius (FLR) effects in plasma physics is well documented (Braginskii 1958; Roberts \& Taylor 1962; Braginskii 1965; Rosenbluth \& Simon 1965; Liley 1972; Callen et al. 1987; Hazeltine \& Meiss 1992; Mikhailovskii 1992; Hazeltine \& Waelbroeck 1998; Sulem \& Passot 2008; Hosking \& Dewar 2016; Goedbloed, Keppens \& Poedts 2019). A broad class of models that incorporate FLR effects are those that fall under the fluid category, i.e. the momenta of the underlying particles are integrated out to yield mean field theories that describe the evolution of physical quantities such as density, fluid velocity, etc. The advantage of the fluid formalism stems from the fact that the complex dynamics of a multiparticle system is reduced to a few dynamical equations that are capable of accurately capturing its essential properties.

Fluid models that include FLR effects are often constructed by incorporating kinetic effects, e.g. by moving from particle phase-space coordinates to guiding centre coordinates (Hasegawa \& Wakatani 1983; Hsu, Hazeltine \& Morrison 1986; Brizard 1992; Smolyakov, Pogutse \& Hirose 1995; Belova 2001); models with FLR contributions incorporate kinetic effects of importance such as Landau damping and gyroradius averaging (Hammett,

$\dagger$ Email address for correspondence: mlingam@fit.edu 
Dorland \& Perkins 1992; Beer \& Hammett 1996; Snyder, Hammett \& Dorland 1997; Waltz et al. 1997; Snyder \& Hammett 2001; Staebler, Kinsey \& Waltz 2005; Madsen 2013). A second approach involves expansions in the smallness of the Larmor radius as compared to a characteristic length scale of the system and the imposition of closures for higher-order moments (Macmahon 1965; Kennel \& Greene 1966; Bowers 1971; Pogutse, Smolyakov \& Hirose 1998; Goswami, Passot \& Sulem 2005; Simakov \& Catto 2006; Ramos 2005a, 2007; Passot \& Sulem 2007; Ramos 2010, 2011; Passot, Sulem \& Hunana 2012; Passot, Sulem \& Tassi 2017; Pfefferlé, Hirvijoki \& Lingam 2017). A third method uses the Hamiltonian framework to construct full and reduced magnetohydrodynamic (MHD) models endowed with FLR and other effects (Morrison \& Hazeltine 1984; Morrison, Caldas \& Tasso 1984; Hsu et al. 1986; Hazeltine, Hsu \& Morrison 1987; Brizard et al. 2008; Tassi et al. 2008; Izacard et al. 2011; Waelbroeck \& Tassi 2012; Comisso et al. 2013; Lingam \& Morrison 2014; Lingam 2015b,c; Passot, Sulem \& Tassi 2018). One of the chief advantages of Hamiltonian methods, as explained in the forthcoming sections, is that they are amenable to the extraction of naturally conserved quantities (the Casimirs) and analysing equilibria and stability.

The Hamiltonian formalism is deeply entwined with its twin approach, building models from an action principle - together, we will refer to them as the Hamiltonian and action principle (HAP) approach. ${ }^{1}$ The HAP formalism has a long history in fluid dynamics and plasma physics - examples of seminal publications prior to the 20th century include Lagrange (1789), Clebsch (1857), von Helmholtz (1858), Clebsch (1859), Hanke (1861) and Kirchhoff (1876). ${ }^{2}$ A summary of modern developments in this area can be found in the reviews by Serrin (1959), Truesdell \& Toupin (1960), Seliger \& Whitham (1968), Arnold (1978), Morrison (1982), Holm et al. (1985), Morrison (1998), Arnold \& Khesin (1998), Morrison (2005), Holm (2008), Morrison (2009), Lingam (2015d), Sudarshan \& Mukunda (2016), Morrison (2017), Tassi (2017) and Webb (2018).

Using the action formalism has many advantages. For a starter, each term in the action has a clear physical meaning, which is not always the case when equations of motion have been derived using phenomenological or ad hoc assumptions. Another advantage is that theories derived from action principles are naturally energy conserving. In some cases, equations of motion that had not been derived using the HAP formalism were erroneously believed to conserve energy (see e.g. Scott 2005, 2007; Kimura \& Morrison 2014; Tronci et al. 2014). In addition, by performing an appropriate Legendre transformation, one can recover the Hamiltonian formalism, which is endowed with several advantages of its own. For a review of action principles in MHD models, we refer the reader to Newcomb (1962), Holm, Marsden \& Ratiu (1998), Morrison (2009), Lingam (2015d), Webb (2018) and for the Hamiltonian formalism to Morrison \& Greene (1980), Morrison (1982), Holm et al. (1985), Morrison (1998, 2005) and Tassi (2017). In particular, we mention its significance in studying symmetric MHD and its properties (Andreussi, Morrison \& Pegoraro 2010, 2012, 2013, 2016) and in constructing and analysing reduced MHD models (Morrison \& Hazeltine 1984; Hazeltine et al. 1987; Kuvshinov, Pegoraro \& Schep 1994; Krommes \& Kolesnikov 2004; Waelbroeck, Hazeltine \& Morrison 2009; Tassi et al. 2010b;

\footnotetext{
${ }^{1}$ We intend this abbreviation to encompass all of the forms of action principles (Hamilton's principle, the phase space action, various constrained variational principles, etc.) and both canonical and noncanonical Hamiltonian descriptions. The HAP approaches of the present paper are Hamilton's principle yielding Lagrange equations, which is here trivially related to the phase space action, the canonical Hamiltonian formulation in the Lagrange variable description, and the noncanonical Eulerian variable description.

${ }^{2}$ Augustin-Louis Cauchy presented a Lagrangian formulation of three-dimensional incompressible hydrodynamics in a seminal, albeit forgotten, work in 1815 (Frisch \& Villone 2014); see also Frisch, Grimberg \& Villone (2017).
} 
Tassi, Grasso \& Pegoraro 2010a; Waelbroeck \& Tassi 2012; Keramidas Charidakos, Waelbroeck \& Morrison 2015; Tassi et al. 2018; Tassi 2019).

Earlier we outlined different methods by which FLR effects can be incorporated into fluid models. It is worth noting that the Hamiltonian methods invoke the use of an interesting device - the gyromap, which was discovered in Morrison et al. (1984) and subsequently employed in the likes of Hazeltine et al. (1987) and Izacard et al. (2011). The gyromap is essentially a noncanonical transformation that maps the phase space to itself, and its chief advantage stems from the fact that it renders the noncanonical bracket of the gyroviscous MHD model identical to that of classical ideal MHD bracket (Morrison \& Greene 1980) when expressed in terms of the new set of noncanonical variables; we will elaborate upon this point later in the paper. ${ }^{3}$ The origin of the gyromap was not properly understood until an action principle analysis in Morrison, Lingam \& Acevedo (2014) was applied to a specific two-dimensional (2-D) model, which assumed a particular ansatz for the internal energy and the gyromap. In this paper, we generalize the work of Morrison et al. (2014) to three dimensions, and present generic results in terms of freely specifiable functions. Furthermore, when we choose a particular ansatz for our FLR fluid model, we will use the physical principles of Larmor gyration to motivate the choice in detail. We will refer to this magnetofluid model as gyroviscous magnetohydrodynamics (GVMHD).

The paper is organized as follows. In $\S 2$, we outline the necessary tools for carrying out an action formulation of three-dimensional (3-D) GVMHD. Then we proceed to build the action in $\S 3$, where we motivate the reasoning behind the gyroviscous term. In $\S 4$, the relevant equations of motion are presented and a particular choice of the gyroviscous ansatz is constructed. In $\S 6$, we present the equivalent Hamiltonian formalism of this model. In $\S 6.2$, we derive the GVMHD bracket and highlight the differences compared with 3-D ideal MHD. Finally, we summarize our results in $\S 7$. Some of the salient auxiliary calculations are presented in the appendices.

\section{The Lagrangian-variable approach to the action principle}

In the first part of this section, we briefly describe Hamilton's principle of stationary action. In the second part, we highlight and outline the Lagrangian picture, and present a systematic methodology for moving to the more commonly used Eulerian picture.

\subsection{Hamilton's principle of stationary action}

The process involved in constructing the action for fluid models has been well known since Lagrange (1789). Once the generalized coordinates $q_{k}(t)$ are chosen, where $k$ runs over all possible degrees of freedom, the action is determined via

$$
S[q]=\int_{t_{0}}^{t_{1}} \mathrm{~d} t L(q, \dot{q}, t),
$$

with $L$ representing the Lagrangian. It must be noted that $S$ is a 'functional', i.e. its domain and range are functions and real numbers, respectively. Hamilton's principle states that that the equations of motion are the extrema of the action, i.e. we require $\delta S[q] / \delta q^{k}=0$, where the functional derivative is defined as follows:

$$
\delta S[q ; \delta q]=\left.\frac{\mathrm{d} S[q+\epsilon \delta q]}{\mathrm{d} \epsilon}\right|_{\epsilon=0}=:\left\langle\frac{\delta S[q]}{\delta q^{i}}, \delta q^{i}\right\rangle .
$$

\footnotetext{
${ }^{3}$ The gyromap is a coordinate change from one set of dynamical variables to another. Its origin and usefulness will be expounded in $\$ 6$.
} 
The continuum version is very similar to the discrete case since the discrete index $k$ is replaced by a continuous one, which we denote by $a$. The coordinate $q$ is a function of $a$ and $t$, and tracks the location of a fluid particle labelled by $a$. We also note the following important quantities which are used throughout the paper: the deformation matrix $\partial q^{i} / \partial a^{j}=: q_{, j}^{i}$ and the corresponding determinant, the Jacobian, $\mathcal{J}:=\operatorname{det}\left(q_{, j}^{i}\right)$. The volume evolves in time via

$$
\mathrm{d}^{3} q=\mathcal{J} \mathrm{d}^{3} a
$$

and the area is governed by

$$
\left(\mathrm{d}^{2} q\right)_{i}=\mathcal{J} a_{, i}^{j}\left(\mathrm{~d}^{2} a\right)_{j},
$$

where $\mathcal{J} a_{, i}^{j}$ is the transpose of the cofactor matrix of $q_{, i}^{j}$. The quantities and the relations introduced above can be used to generate a wide range of identities. One can find a detailed discussion of these, for example, in Serrin (1959), Morrison (1998) and Bennett (2006).

\subsection{Two representations: the Lagrangian and the Eulerian points of view}

The Lagrangian position $q$ evolves in time and is entirely characterized by its label $a$. But the fluid parcels are not solely determined by the position alone; they can also carry with them a certain density, entropy and magnetic field. As the fluid moves along its trajectory, these quantities are also transported along with it, and are consequently characterized only by the label $a$ as well. We will refer to these quantities as attributes. As the label $a$ is independent of time, these attributes serve as Lagrangian constants of motion. The subscript 0 will be used to label the attributes, in order to distinguish them from their Eulerian counterparts.

Let us now consider the Eulerian picture. All Eulerian fields depend on the position $\boldsymbol{r}:=$ $\left(x^{1}, x^{2}, x^{3}\right)$ and time $t$, which can both be measured in the laboratory. As a result, we shall refer to these fields as observables. Moving from the Eulerian to Lagrangian viewpoint and vice versa is accomplished with the Lagrange-Euler maps which we describe below in more detail.

The Eulerian velocity field $\boldsymbol{v}(\boldsymbol{r}, t)$ is the velocity of the fluid element at a location $\boldsymbol{r}$ and time $t$. If we seek to preserve the equivalence of the Lagrangian and Eulerian pictures, this must also equal $\dot{\boldsymbol{q}}(\boldsymbol{a}, t)$. As a result, it is evident that we require $\dot{\boldsymbol{q}}(\boldsymbol{a}, t)=\boldsymbol{v}(\boldsymbol{r}, t)$, where the dot indicates that the time derivative is obtained at fixed label $\boldsymbol{a}$. However, there is a discrepancy since the left-hand side is a function of $\boldsymbol{a}$ and $t$, while the right-hand side involves $\boldsymbol{r}$ and $t$. This conundrum is resolved by noting that the fluid element is at $\boldsymbol{r}$ in the Eulerian picture, and at $\boldsymbol{q}$ in the Lagrangian one. Hence, we note that $\boldsymbol{r}=\boldsymbol{q}(\boldsymbol{a}, t)$, which implies that $\boldsymbol{a}=\boldsymbol{q}^{-1}(\boldsymbol{r}, t)=: \boldsymbol{a}(\boldsymbol{r}, t)$ upon inversion. As a result, our final Lagrange-Euler map for the velocity is

$$
\boldsymbol{v}(\boldsymbol{r}, t)=\left.\dot{q}(a, t)\right|_{a=a(r, t)} .
$$

Now we consider the attributes defined earlier, which we have noted are carried along by the fluid. The first attribute is the entropy of the fluid particle, which we shall label $s_{0}$. For ideal fluids, one expects the entropy to remain constant along the fluid trajectory. In other words, the Eulerian specific entropy $s(\boldsymbol{r}, t)$ must also remain constant throughout, implying that $s=s_{0}$. Apart from entropy, the magnetic stream function $\psi$ for 2-D GVMHD (Andreussi et al. 2013; Morrison et al. 2014) also obeys this property.

Next, we can consider attributes which obey a conservation law similar to the density. The conservation law in this case is that of mass conservation. The attribute is denoted by $\rho_{0}(\boldsymbol{a})$ and the observable by $\rho(\boldsymbol{r}, t)$. The statement of mass conservation in a given (infinitesimal) volume amounts to $\rho(\boldsymbol{r}, t) \mathrm{d}^{3} r=\rho_{0}(\boldsymbol{a}) \mathrm{d}^{3} a$. Using (2.3) we obtain 
$\rho_{0}=\rho \mathcal{J}$. As a result, we have found the Lagrange-Euler map for $\rho$. There exist other attribute-observable pairs in the literature, which also possess similar conservation laws, such as the entropy density.

In the case of magnetofluid models, it is often advantageous to introduce the magnetic field attribute $\boldsymbol{B}_{0}(\boldsymbol{a})$. In the case of ideal magnetofluid models, the conservation law of frozen-in magnetic flux is applicable. In algebraic terms, this amounts to $\boldsymbol{B} \cdot \boldsymbol{d}^{2} \boldsymbol{r}=\boldsymbol{B}_{0}$. $d^{2} a$, and from (2.4) we obtain $\mathcal{J} B^{i}=q_{, j}^{i} B_{0}^{j}$.

In all of the above expressions, the picture is still incomplete since we need to remove the $\boldsymbol{a}$-dependence of the attributes. In a manner similar to that undertaken for the velocity, we evaluate the attributes at $\boldsymbol{a}=\boldsymbol{q}^{-1}(\boldsymbol{r}, t)=: \boldsymbol{a}(\boldsymbol{r}, t)$. This completes our prescription, and one can fully determine the observables once we are provided the attributes in conjunction with the Lagrangian coordinate $q$.

We may also represent the Lagrange-Euler map in an integral form, which permits a more intuitive interpretation. We shall start with the assumption that the attribute-observable relations are found via appropriate conservation laws. We have stated before that one moves from the Lagrangian to the Eulerian picture by 'plucking out' the fluid element that happens to be at the Eulerian observation point $r$ at time $t$. Such a process is accomplished mathematically via the delta function $\delta(\boldsymbol{r}-\boldsymbol{q}(\boldsymbol{a}, t))$. For instance, we see that the density can be treated as follows:

$$
\begin{aligned}
\rho(\boldsymbol{r}, t) & =\int_{D} \mathrm{~d}^{3} a \rho_{0}(\boldsymbol{a}) \delta(\boldsymbol{r}-\boldsymbol{q}(\boldsymbol{a}, t)) \\
& =\left.\frac{\rho_{0}}{\mathcal{J}}\right|_{\boldsymbol{a}=\boldsymbol{a}(\boldsymbol{r}, t)} .
\end{aligned}
$$

Further below, we will also use a new variable, the canonical momentum density $\boldsymbol{M}^{c}=$ $\left(M_{1}^{c}, M_{2}^{c}, M_{3}^{c}\right)$, which is related to its Lagrangian counterpart via

$$
\begin{aligned}
\boldsymbol{M}^{c}(\boldsymbol{r}, t) & =\int_{D} \mathrm{~d}^{3} a \boldsymbol{\Pi}(\boldsymbol{a}, t) \delta(\boldsymbol{r}-\boldsymbol{q}(\boldsymbol{a}, t)) \\
& =\left.\frac{\boldsymbol{\Pi}(\boldsymbol{a}, t)}{\mathcal{J}}\right|_{\boldsymbol{a}=\boldsymbol{a}(\boldsymbol{r}, t)} .
\end{aligned}
$$

For ideal MHD, the canonical momentum density is $\boldsymbol{\Pi}(a, t)=\left(\Pi_{1}, \Pi_{2}, \Pi_{3}\right)=\rho_{0} \dot{\boldsymbol{q}}$. It is worth noting that $\boldsymbol{\Pi}(\boldsymbol{a}, t)$ can be found from the Lagrangian through $\boldsymbol{\Pi}(\boldsymbol{a}, t)=\delta L / \delta \dot{\boldsymbol{q}}$ and does not necessarily equal $\rho_{0} \dot{\boldsymbol{q}}$ in general. One can also construct such integral relations for the entropy and the magnetic field. We refer the reader to Morrison et al. (2014) for a more detailed discussion along these lines.

\section{Action principle for a generic magnetofluid}

The first part of this section is devoted to a brief description of the procedure outlined in Morrison (2009) and Morrison et al. (2014) for constructing action principles for magnetofluid models. Some of the advantages have been highlighted in the introduction, and others can be found in, for example, Morrison (2009) and Morrison et al. (2014). Then, we proceed to construct our action and motivate our choice of terms along the way.

\subsection{The general action}

The domain of integration $D$ is chosen to be a subset of $\mathbb{R}^{3}$. Central to our formulation is the Lagrangian coordinate $q: D \rightarrow D$, which we shall assume to be a well-behaved 
function with the required smoothness, invertibility, etc. Next we need to specify our set of observables, or alternatively our set of attributes. For our models, we work with $\mathfrak{E}=$ $\{\boldsymbol{v}, \rho, \sigma, \boldsymbol{B}\}$ where $\sigma=\rho s$ is the entropy density. Finally, we shall impose the Eulerian closure principle, which is necessary for our model to be 'Eulerianizable'. Mathematically, this principle amounts to the action being fully expressible in terms of the Eulerian observables. Physically, the principle states that our theory must be solely describable in terms of physically meaningful quantities, the observables, and must also give rise to equations of motion in terms of these observables. As a result, we require our action to be given via

$$
S[\boldsymbol{q}]:=\int_{T} \mathrm{~d} t \int_{D} \mathrm{~d}^{3} a \mathcal{L}(\boldsymbol{q}, \dot{\boldsymbol{q}}, \partial \boldsymbol{q} / \partial \boldsymbol{a})=: \bar{S}[\mathfrak{E}] .
$$

As per the Eulerian closure principle, this amounts to finding an action $\bar{S}=\int_{\mathcal{T}} \mathrm{d} t \int_{D} \mathrm{~d}^{3} r \overline{\mathcal{L}}$ in terms of the Eulerian observables. The presence of the bar indicates that the action and the Lagrangian density are expressed solely in terms of the observables.

\subsection{Constructing the gyroviscous action}

The first step in the process involves the construction of the kinetic energy, which must also satisfy the closure principle. Using the analogy with particle mechanics, we know that it equals

$$
S_{\text {kin }}:=\int_{\mathcal{T}} \mathrm{d} t \int_{D} \mathrm{~d}^{3} a \frac{1}{2} \rho_{0}|\dot{\boldsymbol{q}}|^{2}=\int_{\mathcal{T}} \mathrm{d} t \int_{D} \mathrm{~d}^{3} r \frac{1}{2} \rho|\boldsymbol{v}|^{2},
$$

where the last equality is obtained by using relations outlined in $\S 2.2$.

The internal energy per unit mass is a function of the entropy density and the density, and in Eulerian terms it can be represented by $U(\rho, \sigma)$. Using the inverse Lagrange-Euler maps, we can construct the Lagrangian internal energy density accordingly,

$$
\begin{aligned}
S_{\text {int }} & :=\int_{\mathcal{T}} \mathrm{d} t \int_{D} \mathrm{~d}^{3} a \rho_{0} U\left(\frac{\rho_{0}}{\mathcal{J}}, \frac{\sigma_{0}}{\mathcal{J}}\right) \\
& =\int_{\mathcal{T}} \mathrm{d} t \int_{D} \mathrm{~d}^{3} r \rho U(\rho, \sigma) .
\end{aligned}
$$

The next step is the construction of the magnetic energy, and we use the same process outlined for the internal energy, viz. we determine the Eulerian term and obtain the Lagrangian version consequently through the Lagrange-Euler map,

$$
\begin{aligned}
S_{\text {mag }} & :=\int_{\mathcal{T}} \int_{D} \mathrm{~d}^{3} r \frac{1}{2}|\boldsymbol{B}|^{2}, \\
& =\int_{\mathcal{T}} \mathrm{d} t \int_{D} \mathrm{~d}^{3} a \frac{1}{2 \mathcal{J}} q_{, j}^{i} q_{, k}^{i} B_{0}^{j} B_{0}^{k} .
\end{aligned}
$$

The magnetic energy is actually $|B|^{2} / 8 \pi$ in CGS units but we drop the factor of $4 \pi$ henceforth by scaling it away through the adoption of Alfvénic units.

Now we are ready to construct the most important term which will be responsible for the gyroviscosity. The gyroviscous term is taken to be linear in $\dot{\boldsymbol{q}}$ and is given by

$$
S_{\text {gyro }}=\int_{\mathcal{T}} \mathrm{d} t \int_{D} \mathrm{~d}^{3} a \dot{\boldsymbol{q}} \cdot \boldsymbol{\Pi}^{\star}=\int_{\mathcal{T}} \mathrm{d} t \int_{D} \mathrm{~d}^{3} r \boldsymbol{v} \cdot \boldsymbol{M}^{\star} .
$$

In other words, we operate under the premise that $\boldsymbol{\Pi}^{\star}$ is solely a functional of $\boldsymbol{q}$ and $t$. As the Eulerian perspective is inherently endowed with physical variables (e.g. density and 
magnetic field), we will focus on the Eulerian equivalent of $\boldsymbol{\Pi}^{\star}$; from the Eulerian closure principle we obtain the relation

$$
M^{\star}=\left.\frac{\Pi^{\star}(a, t)}{\mathcal{J}}\right|_{a=a(r, t)} .
$$

The complete action functional is now given by

$$
S=S_{\text {kin }}-S_{\text {int }}-S_{\text {mag }}+S_{\text {gyro }} .
$$

The action of (3.7) is general, but not the most general second-order (in $v$ ) action that satisfies the Eulerian closure principle. For example, the term $S_{\text {kin }}$ could be generalized by replacing its integrand with $\rho_{0} G|\dot{\boldsymbol{q}}|^{2} /\left.2\right|_{\boldsymbol{a}}=\rho G(\rho, \sigma, \boldsymbol{B})|\boldsymbol{v}|^{2} / 2$ and the integrand of $S_{\text {int }}$ could be replaced by $\left.\rho_{0} U\right|_{\boldsymbol{a}}=\rho U(\rho, \sigma, \boldsymbol{B})$, a form that was shown in Morrison (1982) to allow for anisotropic pressure. Here both $G$ and $U$ could be arbitrary functionals (including derivatives) of their arguments. Similarly the term $S_{\text {mag }}$ could be generalized.

The Eulerian canonical momentum density is defined via (2.7), which can be computed by finding the Lagrangian canonical momentum using $\boldsymbol{\Pi}(\boldsymbol{a}, t)=\delta L / \delta \dot{\boldsymbol{q}}$ and Eulerianizing it. Upon doing so, we arrive at the so-called gyromap, a device introduced in Morrison et al. (1984) as follows:

$$
\boldsymbol{M}^{c}:=\rho \boldsymbol{v}+\boldsymbol{M}^{\star}=\boldsymbol{M}+\boldsymbol{M}^{\star}
$$

The benefit of employing the gyromap and its natural origin will be discussed in $\S 6$ and further explicated in appendix B.

So far we have only required $\boldsymbol{M}^{\star}$ to satisfy the closure principle, i.e. that it be expressible in terms of the subset $\{\rho, \sigma, \boldsymbol{B}\} \subset \mathfrak{E}$, including all possible Eulerian derivatives. Given that $\boldsymbol{M}^{\star}$ is a momentum density, arising perhaps from underlying gyration of particles, a natural assumption is that it has the magnetization form

$$
M^{\star}=\nabla \times L^{\star}
$$

i.e. we assume that $\boldsymbol{M}^{\star}$ is divergence-free. Since we are interested in a gyroviscosity due to gyromotion, this is a physically reasonable assumption. However, one could replace (3.9) by a Helmholtz decomposition for a more general collisionless viscosity. The present choice is also motivated in part by the realization in Morrison et al. (1984) and Morrison et al. (2014) that this choice is consistent with existing 2-D gyroviscous models. Because $\boldsymbol{M}^{\star}$ has the units of momentum density, from which we see that the quantity $\boldsymbol{J}^{\star} \propto$ $(q / m) M^{\star}$ resembles a current density. If one assumes that the fluid 'particles' possess a finite magnetic moment, it follows that the fluid must have a finite magnetization. In other words, one may identify $J^{\star}$ with the magnetization current density, which is divergence-free (Jackson 1998) and the current through an area depends on flux through a bounding curve. Are other choices possible and do any of them conserve angular momentum? Perhaps an even simpler way of envisioning the ansatz for $\boldsymbol{M}^{\star}$ is that it must emerge from the gyration of particles. In pictorial terms, this gyration is reminiscent of the effect generated by the curl of a vector field, which motivates our choice of $\boldsymbol{M}^{\star}$. Further grounds for assuming this particular expression are described in Morrison et al. $(2014, \S 5)$. With this ansatz, evidently $\nabla \cdot \boldsymbol{M}^{c}=\boldsymbol{\nabla} \cdot \boldsymbol{M}$, since the second term vanishes. Note that the right-hand side of this expression appears in the continuity equation, and we see that one could also replace it by the left-hand side if we operate with $M^{\star}=\nabla \times L^{\star}$. Furthermore, dimensional analysis permits the identification of $L^{\star}$ with the angular momentum density.

As we have reduced the question of determining $L^{\star}$, we must ask ourselves as to whether any further simplifications are feasible. Once again, we can resort to physical intuition 
to gain an idea of what $\boldsymbol{L}^{\star}$ might look like. Without further special assumptions about the fluid, e.g. it having some intrinsic or extrinsic direction, the vectorial character of $L^{\star}$ must come from $B$ or from the set of gradients of the observables; these and their cross products are the only vectors available. Thus, for example, a general form for $L^{\star}$ could be composed of a linear combination of these vectors with coefficients dependent on $\rho, \sigma$ and $|\boldsymbol{B}|$. If we assume $\boldsymbol{L}^{\star}$ constitutes an internal angular momentum density of some kind associated with particle gyration, then it is reasonable to posit that it would tend to align with the magnetic field $\boldsymbol{B}$. Moreover, in the limit of a large magnetic field, the corresponding gyroradii would become small, owing to which the fluid particle may not be significantly affected by gradients on these scales. Combining the preceding arguments leads to the generic form

$$
\boldsymbol{L}^{\star}=\mathcal{F}(\rho, \sigma,|\boldsymbol{B}|) \boldsymbol{B}
$$

In $\S 4.2$ we will argue for further specification of the properties of (3.10).

With the choice of (3.10), the gyroviscous term of the action, expressed in terms of the observables is given by

$$
\begin{aligned}
S_{\text {gyro }} & =\int_{\mathcal{T}} \mathrm{d} t \int_{D} \mathrm{~d}^{3} r \boldsymbol{v} \cdot \nabla \times[\boldsymbol{B \mathcal { F }}(\rho, \sigma,|\boldsymbol{B}|)] \\
& =\int_{\mathcal{T}} \mathrm{d} t \int_{D} \mathrm{~d}^{3} r \mathcal{F} \boldsymbol{B} \cdot \nabla \times \boldsymbol{v},
\end{aligned}
$$

where the second equality follows from integrating by parts and neglecting the boundary term. We shall use the latter operation consistently throughout the rest of the paper. Now that we have constructed the gyroviscous term, we note that it is still generic since there is considerable freedom in the choice of $\mathcal{F}$.

\section{The equations of motion and the choice of ansatz}

In this section, we shall present the equations of motion and discuss the origin of the gyroviscous terms, and why a specific choice of the free function $\mathcal{F}$ emerges in a natural manner.

\subsection{The equations of motion}

The equations for the density, entropy density and the magnetic field can be determined via the attributes/observables relations defined through the appropriate conservation laws and the Lagrange-Euler maps. The entropy density and the density obey similar laws, given by

$$
\begin{aligned}
& \frac{\partial \rho}{\partial t}+\nabla \cdot(\rho \boldsymbol{v})=0, \\
& \frac{\partial \sigma}{\partial t}+\nabla \cdot(\sigma \boldsymbol{v})=0 .
\end{aligned}
$$

The equation governing the magnetic field is

$$
\frac{\partial \boldsymbol{B}}{\partial t}+\boldsymbol{B}(\boldsymbol{\nabla} \cdot \boldsymbol{v})-(\boldsymbol{B} \cdot \nabla) \boldsymbol{v}+(\boldsymbol{v} \cdot \nabla) \boldsymbol{B}=0,
$$

which can be recast into the more familiar induction equation if $\nabla \cdot B=0$ is satisfied. If the constraint is obeyed, then we obtain

$$
\frac{\partial \boldsymbol{B}}{\partial t}=\nabla \times(\boldsymbol{v} \times \boldsymbol{B}) .
$$


The dynamical equation for the momentum is derived from $\delta S=0$, and is thus equal to

$$
\begin{aligned}
& \frac{\partial}{\partial t}\left(\rho v^{k}\right)+\partial_{j}\left[\rho v^{j} v^{k}+\left(p+\frac{|\boldsymbol{B}|^{2}}{2}\right) \delta^{j k}-B^{j} B^{k}\right] \\
& -\partial_{j}\left[\boldsymbol{B} \cdot(\nabla \times \boldsymbol{v})\left(\rho \frac{\partial \mathcal{F}}{\partial \rho}+\sigma \frac{\partial \mathcal{F}}{\partial \sigma}+|\boldsymbol{B}| \frac{\partial \mathcal{F}}{\partial|\boldsymbol{B}|}-\mathcal{F}\right) \delta^{j k}\right] \\
& +\partial_{j}\left[\boldsymbol{B} \cdot(\nabla \times \boldsymbol{v})\left(\frac{\partial \mathcal{F}}{\partial|\boldsymbol{B}|} \frac{B^{j} B^{k}}{|\boldsymbol{B}|}\right)\right] \\
& +\partial_{j}\left[\epsilon_{k j i} B^{i}\left(\frac{\partial \mathcal{F}}{\partial \rho} \frac{\partial \rho}{\partial t}+\frac{\partial \mathcal{F}}{\partial \sigma} \frac{\partial \sigma}{\partial t}+\frac{\partial \mathcal{F}}{\partial|\boldsymbol{B}|} \frac{\boldsymbol{B}}{|\boldsymbol{B}|} \cdot \frac{\partial \boldsymbol{B}}{\partial t}\right)\right]+\partial_{j}\left[\epsilon_{k j i} \mathcal{F} \frac{\partial B^{i}}{\partial t}\right] \\
& -\partial_{j}\left[\epsilon_{l j i} \mathcal{F} B^{l}\left(\partial_{k} v^{i}\right)-\epsilon_{k l i} \mathcal{F} B^{j}\left(\partial_{l} v^{i}\right)\right]=0,
\end{aligned}
$$

where repeated indices indicate summation (as per the Einstein convention), and we have employed the standard relationship between the internal energy and the scalar pressure $p$. We note that (4.5) can be obtained in two different ways from the action. The first is to follow the conventional variation with respect to $q$ and obtain it accordingly. The second method involves the use of the procedure outlined in Frieman \& Rotenberg (1960) and Newcomb (1962) and is described in appendix A. For our model, (4.1)-(4.3) and (4.5) constitute the complete set of dynamical equations.

Before discussing the ansatz in more detail, a few observations regarding (4.5) are in order. The second term occurring in the first line of this equation represents the ideal MHD momentum flux (enclosed in square brackets), which is seen from the absence of $\mathcal{F}$ in it. The second and third lines contain terms that are purely symmetric under the interchange $k \leftrightarrow j$. The fourth line contains terms that are wholly antisymmetric under $k \leftrightarrow j$. The fifth (and final) line contains terms that are neither purely symmetric nor purely antisymmetric. As a result, we see that the entire momentum flux tensor is not symmetric, as opposed to the ideal MHD tensor, or the 2-D gyroviscous tensor for the specific model considered in Morrison et al. (2014). Note that we refer to the terms from line two onwards as gyroviscous because they are expressed in terms of the velocity shear, akin to viscous hydrodynamics. The gyroviscous tensor thus obtained above can be compared against the general expression(s) presented in Ramos (2005b). Furthermore, these effects arise from charged particle gyration - the latter aspect is explored below.

\subsection{The origin of the gyroviscous ansatz}

In $\S 3.2$, we briefly outlined the process involved in constructing a generic gyroviscous term. Now, we shall draw upon further physics to select a specific choice for the ansatz.

First, let us suppose that we start out with the notion of an internal angular momentum $L^{\star}$. In order to understand where this angular momentum originates, we recall an identity from electromagnetism which relates the angular momentum to the magnetic moment via the gyromagnetic ratio, $(2 \mathrm{~m}) / e$. If we consider a two-species model of ions and electrons, then the ions will play the dominant role, owing to their higher mass. Hence, we know that $L^{\star}=(2 m / e) \boldsymbol{\mu}$. The magnetic moment $\boldsymbol{\mu}$ is typically an adiabatic invariant in plasmas, and its magnitude is given by $|\boldsymbol{\mu}|=m \boldsymbol{v}_{\perp}^{2} / 2|\boldsymbol{B}|$, which is proportional to $P_{\perp} /|\boldsymbol{B}|$ where $P_{\perp}$ denotes the perpendicular component of the (anisotropic) pressure. But, the magnetic moment is a vector and the most natural way to construct a vector is through the unit vector of the magnetic field. Putting these results together, we find that a natural ansatz (albeit a 
specific one) for $L^{\star}$ is given by

$$
\boldsymbol{L}^{\star}=\alpha \frac{m}{2 e} \frac{P_{\perp}}{|\boldsymbol{B}|^{2}} \boldsymbol{B},
$$

where $\alpha$ is a dimensionless proportionality constant, which can be arbitrarily specified; in the ensuing analysis, we set $\alpha=1$ for simplicity. By comparison with the more general ansatz outlined in $\S 3.2$, we find that they are identical when $\mathcal{F}=\alpha(m / 2 e) P_{\perp} /|\boldsymbol{B}|^{2}$.

The function $P_{\perp}$ is a function of $\sigma, \rho$ and $|\boldsymbol{B}|$. For a more detailed discussion of the anisotropic pressure, we refer the reader to Kimura \& Morrison (2014). It is defined as

$$
P_{\perp}=\rho^{2} \frac{\partial U}{\partial \rho}+\rho|\boldsymbol{B}| \frac{\partial U}{\partial|\boldsymbol{B}|},
$$

an expression that first appeared in Morrison (1982), where $U$ is the internal energy that is a function of $\rho$ and $\sigma$, but also of the magnetic field; see also Hazeltine, Mahajan \& Morrison (2013). If we wish to forgo anisotropy, then we assume that $U$ is independent of $B$, and hence the second term in the above term vanishes. This assumption was used in deriving the equation of motion (4.5) since the internal energy introduced in (3.3) had no $\boldsymbol{B}$-dependence. Such an assumption also leads to the pressure tensor becoming isotropic, given by the first term of (4.7) alone.

In summary, the ansatz constructed was chosen such that the gyroviscosity (and consequently the momentum transport) arises via the gyration of charged particles, thereby lending the term its name. The fact that momentum transport could take place via such gyrations was first noted by Chapman \& Cowling (1970) and Kaufman (1960) in the 1950s and 1960s. This principle was applied to incompressible gyrofluids in Newcomb (1972, 1973, 1983) and compressible gyrofluids in Morrison (2009) and Morrison et al. (2014), who showed that this specific ansatz yielded results that were fully compatible with the 2-D version of the Braginskii tensor (Braginskii 1965).

Lastly, we note that substituting (4.6) in (3.8) after employing $M^{\star}=\nabla \times L^{\star}$ will yield a number of extra terms with the same dimensions as $\boldsymbol{M}=\rho \boldsymbol{v}$. Hence, if one divides the expression throughout by $\rho$, the contributions arising from $\boldsymbol{M}^{\star}$ have the dimensions of velocity and possess physical interpretations. The first term, which is proportional to $\left(\boldsymbol{B} \times \nabla P_{\perp}\right) /|\boldsymbol{B}|^{2}$, amounts to the diamagnetic drift velocity. The second term, which is proportional to $P /|\boldsymbol{B}|^{3}(B \times \nabla|\boldsymbol{B}|)$ is analogous to the $\nabla|\boldsymbol{B}|$ drift velocity for charged particles. This correspondence has been pointed out in Morrison et al. $(1984, \S 6)$.

\section{Angular momentum conservation and its ramifications}

In this section, we discuss the chief unusual property of our model - the lack of an 'orthodox' angular momentum conservation, and its resolution. We also present a brief illustration of its ramifications in an astrophysical context.

\subsection{Constructing a hybrid conserved angular momentum}

When we perform the constrained variation of our action, we recover

$$
\left.\begin{array}{c}
\frac{\partial M_{i}^{c}}{\partial t}+\partial_{j} T_{i j}=0, \\
T_{i j}=M_{i}^{c} v_{j}-\frac{\partial \mathcal{L}}{\partial\left(\partial_{j} v_{k}\right)}\left(\partial_{i} v_{k}\right)+\frac{\partial \mathcal{L}}{\partial B^{i}} B_{j}+\delta_{i j}\left[\mathcal{L}-\frac{\partial \mathcal{L}}{\partial B^{k}} B^{k}-\frac{\partial \mathcal{L}}{\partial \rho} \rho\right] .
\end{array}\right\}
$$

Additional details can be found in Holm et al. (1998, equations (7.6)-(7.8)) and Lingam $\&$ Morrison $(2014, \S 3)$. Note that the Lagrangian density $\mathcal{L}$ in the above expression 
refers to the one present in (3.7). A rather unusual fact emerges if one inspects the above energy-momentum tensor: when one considers ideal MHD, or even Hall and extended MHD, the tensor $T_{i j}$ is symmetric. In turn, this ensures that the angular momentum $\boldsymbol{M}=\boldsymbol{r} \times \rho \boldsymbol{v}$ is conserved. However, this is evidently not the case for the above energy-momentum tensor.

This fact is not unusual because a number of hydrodynamic models are known to possess asymmetric energy-momentum tensors. In particular, if the constituent 'particles' (which may be fluid parcels) have an internal degree of freedom (i.e. spin), the energy-momentum tensor of the fluid will manifest a non-symmetric component (Papapetrou 1949; Snider \& Lewchuk 1967; Olmsted \& Snider 1976; Dewar 1977; Evans 1979; Kopczyński 1990; Lingam 2015a). Examples of hydrodynamic models with asymmetric energy-momentum tensors include ferrohydrodynamics (Rosensweig 1985; Billig 2005) and nematics (de Gennes \& Prost 1993). Although many core plasma models are characterized by symmetric energy-momentum tensors (Pfirsch \& Morrison 1985; Similon 1985), other plasma models feature asymmetric energy-momentum tensors (e.g. Brizard 2010a). In consequence, not all components of the angular momentum will be conserved, although the toroidal component is conserved in such models (Scott \& Smirnov 2010).

To resolve this, we will adopt the procedure delineated in McLennan (1966). We begin with the observation that the first expression in (5.1) remains invariant under the transformations $M_{i}^{c} \rightarrow M_{i}^{c}+\partial_{j} \Sigma_{i j}$ and $T_{i j} \rightarrow T_{i j}-\partial \Sigma_{i j} / \partial t$. Let us suppose that we choose $\partial \Sigma_{i j} / \partial t$ to be the antisymmetric part of $T_{i j}$, thereby ensuring that $T_{i j}-\partial \Sigma_{i j} / \partial t$ is purely symmetric. Hence, by utilizing this choice of $\Sigma_{i j}$, we find that

$$
\frac{\partial \Sigma_{i j}}{\partial t}=T_{i j}^{A}=\frac{1}{2}\left(T_{i j}-T_{j i}\right)=\epsilon_{i j k} \tau_{k},
$$

where $\tau$ has the units of torque density and is given by

$$
\tau_{k}=\frac{1}{2}\left[\epsilon_{k a b} M_{a}^{c} v_{b}+\frac{m}{2 e} \frac{P_{\perp}}{|B|^{2}}\left(B_{k} \partial_{l} v_{l}-B_{l} \partial_{k} v_{l}\right)\right] .
$$

The first term in the above expression is $\boldsymbol{M}^{c} \times \boldsymbol{v}$, which can also be expressed as $\boldsymbol{M}^{\star} \times \boldsymbol{v}$ since $\rho \boldsymbol{v} \times \boldsymbol{v}=0$. The second and third terms are proportional to $(\boldsymbol{\nabla} \cdot \boldsymbol{v}) \boldsymbol{B}$ and $(\boldsymbol{\nabla} \boldsymbol{v}) \cdot \boldsymbol{B}$, respectively. Since we know that $\tau$ behaves as a torque density, let us define a dynamical variable $\mathcal{S}$ such that $\partial \mathcal{S}_{k} / \partial t=\tau_{k}$; this constitutes a relation that mirrors the conventional torque-angular momentum relation in classical mechanics. Using this in (5.2), we find that $\Sigma_{i j}=\epsilon_{i j k} \mathcal{S}_{k}$. With these ingredients, we can now construct a symmetric momentum conservation law as follows:

$$
\frac{\partial M_{i}^{\mathrm{tot}}}{\partial t}+\partial_{j} T_{i j}^{S}=0,
$$

with $T_{i j}^{S}$ representing the symmetric energy-momentum tensor and $M_{i}^{\text {tot }}=M_{i}^{c}+\epsilon_{i j k} \partial_{j} \mathcal{S}_{k}$. As the resultant energy-momentum tensor is symmetric, it follows that the corresponding angular momentum $\boldsymbol{r} \times \boldsymbol{M}^{\text {tot }}$ is conserved.

The ramifications of $\mathcal{S}$ are manifold. It can be interpreted as an intrinsic angular momentum density generated from the torque density (5.3). This is consistent with prior works (Papapetrou 1949; Snider \& Lewchuk 1967; Olmsted \& Snider 1976; Dewar 1977; Evans 1979; Kopczyński 1990) that outlined the connections between intrinsic angular momentum and a non-symmetric energy-momentum tensor. A second justification arises from $\boldsymbol{M}^{\text {tot }}=\boldsymbol{M}^{c}+\nabla \times \mathcal{S}$, implying by dimensional analysis that $\mathcal{S}$ has the dimensions of angular momentum density. If we define $\boldsymbol{M}^{\text {int }}=\nabla \times \mathcal{S}$, we see that $\nabla \cdot \boldsymbol{M}^{\text {int }}=0$. 
The kinship between $\boldsymbol{M}^{\star}$ and $\boldsymbol{M}^{\text {int }}$ is obvious as they are both generated via an internal angular momentum mechanism and are divergence-free.

Let us now summarize our results. We defined a dynamical variable $\mathcal{S}$ such that it obeys $\partial \mathcal{S}_{i} / \partial t=\tau_{i}$ where $\tau$ is given by (5.3), and it emerges from the antisymmetric part of the original energy-momentum tensor. We also find that the new momentum $\boldsymbol{M}^{\text {tot }}=\boldsymbol{M}+$ $\left(\boldsymbol{M}^{\star}+\boldsymbol{M}^{\text {int }}\right.$ ) yields a symmetric momentum tensor (which is the symmetric part of the old one). Using the expressions for $\boldsymbol{M}^{\star}$ and $\boldsymbol{M}^{\text {int }}$, we have

$$
M^{\star}+M^{\text {int }}=\nabla \times\left(L^{\star}+\mathcal{S}\right) .
$$

Hence, we can define a composite intrinsic angular momentum $J=L^{\star}+\mathcal{S}$, akin to the total angular momentum in quantum mechanics (Weinberg 2015). The introduction of $\boldsymbol{J}$ yields $\boldsymbol{M}^{\text {tot }}=\boldsymbol{M}+\nabla \times \boldsymbol{J}$, which is simple in form and has an immediate physical interpretation. The angular momentum corresponding to $\boldsymbol{M}^{\text {tot }}$ is conserved, and is given by $\boldsymbol{r} \times \boldsymbol{M}^{\text {tot }}$. Hence, the total angular momentum defined below is an invariant,

$$
\int \mathrm{d}^{3} r[\boldsymbol{r} \times \boldsymbol{M}+\boldsymbol{r} \times(\nabla \times J)] .
$$

Before proceeding further, some major aspects concerning the 2-D GVMHD model described in Morrison et al. (1984) and Morrison et al. (2014) merit further explication. To begin with, we can rewrite (5.1) as follows:

$$
\frac{\partial M_{i}}{\partial t}+\partial_{j} \tilde{T}_{i j}+\left[\frac{\partial M_{i}^{\star}}{\partial t}+\partial_{j}\left(M_{i}^{\star} v_{j}\right)\right]=0,
$$

where we have introduced the new energy-momentum tensor

$$
\tilde{T}_{i j}=\rho v_{i} v_{j}-\frac{\partial \mathcal{L}}{\partial\left(\partial_{j} v_{k}\right)}\left(\partial_{i} v_{k}\right)+\frac{\partial \mathcal{L}}{\partial B_{i}} B_{j}+\delta_{i j}\left[\mathcal{L}-\frac{\partial \mathcal{L}}{\partial B_{k}} B_{k}-\frac{\partial \mathcal{L}}{\partial \rho} . \rho\right] .
$$

The first key point worth highlighting here is that Morrison et al. (1984, 2014) adopted: (i) a specific equation of state (EOS) for $P_{\perp}$ wherein $P_{\perp} /|\boldsymbol{B}|$ was a Lie-dragged scalar density; and (ii) the choice $B=B_{z} \hat{z}$ for the magnetic field. These two conditions collectively ensured that $L^{\star}$ had only one component and that the components of $\boldsymbol{M}^{\star}$ behaved as scalar densities that underwent Lie-dragging; in other words, the term inside the square brackets of (5.7) vanishes identically for the 2-D GVMHD model.

The second essential point is that 2-D GVMHD did not include any variables that were Lie-dragged as vector densities of rank unity. In contrast, the magnetic field in 3-D MHD and GVMHD plays this role (Morrison 1982; Lingam \& Morrison 2014), ${ }^{4}$ but $B_{z}$ in 2-D GVMHD is a Lie-dragged scalar density as seen from Morrison et al. (1984, equation (3)); to put it differently, $B_{z}$ in 2-D GVMHD is advected the same way as the plasma density $\rho$. Thus, the terms in (5.8) involving $B_{i}$ are rendered irrelevant because they were derived under the assumption that the magnetic field is a Lie-dragged vector density. Hence, these two facts collectively ensure that the only potential source of asymmetry in the energy-momentum tensor of 2-D GVMHD is the second term on the right-hand side of (5.8). When one utilizes the particular EOS for this model in conjunction with

\footnotetext{
${ }^{4}$ Alternatively, if one considers the Hodge dual of the magnetic field, it constitutes an example of a Lie-dragged two-form (Tur \& Yanovsky 1993).
} 
$M_{z}=0$ and $\boldsymbol{B}=B_{z} \hat{z}$, it can be shown (Morrison et al. 1984, 2014) that the gyroviscous term of 2-D GVMHD yields the contribution

$$
T_{i j}^{(2 \mathrm{D}-\mathrm{GV})}=\frac{m}{2 e} \frac{P_{\perp}}{B_{z}} \mathcal{N}_{j l i k} \partial_{k} v_{l}, \quad \mathcal{N}_{j l i k}=\delta_{j k} \epsilon_{l i}-\delta_{l i} \epsilon_{j k},
$$

to the energy-momentum tensor, which turns out to be fully symmetric.

The above discussion serves to illustrate how and why the energy-momentum tensor of the simplified 2-D GVMHD model of Morrison et al. $(1984,2014)$ is symmetric in nature. However, in order to achieve this symmetry, a number of restrictions on the EOS as well as the magnetic field and momentum density had to be imposed. When all of these constraints are relaxed, which is the case for 3-D GVMHD, one finds that an asymmetric energy-momentum tensor is obtained.

\subsection{An illustration of the formalism}

We have already noted earlier that the kinetic angular momentum $r \times M$ is not conserved. However, we have seen that the angular momentum described in (5.6) is conserved. Together, these imply that the rate of loss (or gain) of the kinetic angular momentum $r \times M$ is precisely equal to the rate of gain (or loss) of the intrinsic angular momentum $J$. Let us recall that $\mathcal{S}$ comprises a part of $\boldsymbol{J}$, and we know that $\partial \mathcal{S}_{i} / \partial t=\tau_{i}$ where $\boldsymbol{\tau}$ is given by (5.3). The first term in (5.3) reduces to $\boldsymbol{M}^{\star} \times \boldsymbol{v}$, as noted earlier. It is worth mentioning that the additional two terms are quite different, but exhibit a similar scaling. Hence, we shall use only the first term in our subsequent analysis. The total torque (denoted by $\tilde{\mathcal{T}}$ ) is found by integrating this term over the volume, and thus gives rise to the scaling

$$
\tilde{\mathcal{T}}=\int \boldsymbol{M}^{\star} \times \boldsymbol{v} \mathrm{d}^{3} r \sim \frac{m}{e}\left(\frac{P_{\perp}}{|\boldsymbol{B}|}\right) \Omega R^{3},
$$

where we have dropped the numerical factors and used a characteristic velocity of $\Omega R$, with $R$ denoting the radius of the (spherical) object. It is evident that the scaling will be entirely determined by the EOS that is adopted.

Next, let us evaluate the spin-down rate, by using the relation $\tilde{\mathcal{T}}=I \dot{\Omega}$, from classical mechanics. The moment of inertia, dropping all numerical factors, is approximately $M R^{2} \sim \rho R^{5}$. Using this in (5.10), we find that

$$
\dot{\Omega} \sim \frac{m}{e}\left(\frac{P_{\perp}}{\rho|\boldsymbol{B}|}\right) \Omega R^{-2} .
$$

The above relation indicates that $\dot{\Omega} \propto \Omega$ (holding other quantities fixed). The EOS depends only on $\rho$, s and $|\boldsymbol{B}|$ and hence we can conclude that the relation $\dot{\Omega} \propto \Omega$ is likely to be independent of the choice of the EOS. If we treat $\rho$ and $R$ to be independent variables, i.e. by choosing $M$ to be the dependent variable, one can also conclude that $\dot{\Omega} \propto R^{-2}$ will be independent of the EOS. The characteristic time $t_{c}=\Omega / \dot{\Omega}$, is expected to be independent of $\Omega$ and is given by

$$
t_{c} \sim \frac{e}{m}\left(\frac{\rho|B|}{P_{\perp}}\right) R^{2}
$$

and we see that it is proportional to $R^{2}$, when the other parameters are held constant. The Chew-Goldberger-Low EOS for $P_{\perp}$ (Chew, Goldberger \& Low 1956) is of particular 
interest since the characteristic time $t_{c}$ and the rate $\dot{\Omega}$ are both independent of the density and the magnetic field, thereby demonstrating an unexpected universality. The resulting spin-down corresponds to the dissipation of kinetic angular momentum, which must imply that there is a corresponding increase in the intrinsic angular momentum $J$ (which comprises the other fluid variables).

The spin rates of low-mass stars are found to slow down by approximately two orders of magnitude over a span of $10^{9}$ years (Scholz 2009). Modelling stellar spin-down is important for a multitude of reasons, including the fact that the older stars (with lower rotation rates) display lower activity in general, which has numerous ramifications for planetary habitability (Lingam \& Loeb 2018, 2019). We can estimate the characteristic time by choosing solar parameters (i.e. a solar-type star) for an order-of-magnitude calculation. In particular, we substitute $|B| \sim 10^{-4} \mathrm{~T}, R \sim 7 \times 10^{8} \mathrm{~m}$ and $T \sim 5.8 \times 10^{3}$ $\mathrm{K}$ (Priest 2014) in (5.12), which yields $t_{c} \sim 3 \times 10^{6}$ years. The two leading candidates invoked to explain stellar spin-down, star-disk and stellar wind braking, operate on time scales of $\sim 10^{6}-10^{7}$ years and $\sim 10^{8}$ years, respectively (Bouvier et al. 2014, $\S 4.1$ ). Hence, we see that our semiquantitative estimate is comparable to these two time scales, and may therefore constitute a viable mechanism for governing angular momentum evolution of solar-mass stars.

The issue of angular momentum losses in protostars is another closely related topic (Bodenheimer 1995; Matt \& Pudritz 2005; Hartmann, Herczeg \& Calvet 2016) which might also be resolvable through the same mechanism. We emphasize that the heuristic treatment in this subsection has primarily relied on simple scaling arguments, and a complete picture can only emerge through the synthesis of rigorous analytical models and numerical simulations. We note that this only represents the tip of the iceberg - other potential applications include pulsar braking, transport in accretion discs and associated phenomena. In the realm of fusion, we note that the formalism developed herein may prove to be useful in explaining intrinsic rotation observed in tokamaks (Gürcan et al. 2007; de Grassie 2009; Diamond et al. 2013; Rice 2016).

\section{The Hamiltonian description and the origin of the gyromap}

In this section, we shall outline some of the basic principles underlying noncanonical Hamiltonian dynamics. The literature on this subject is considerable, and we refer the reader to Morrison (1998) for a comprehensive introduction.

\subsection{The Lagrangian view point and the Lagrange-Euler map}

First, note that the Hamiltonian can be obtained from the Lagrangian via a Legendre transform, akin to the usual process in particle mechanics. The Hamiltonian is given by

$$
H[\boldsymbol{q}, \boldsymbol{\Pi}]=\int_{D} \mathrm{~d}^{3} a \dot{\boldsymbol{q}} \cdot \boldsymbol{\Pi}-L,
$$

where

$$
L[\dot{\boldsymbol{q}}, \boldsymbol{q}]=\int_{D} \mathrm{~d}^{3} a \mathcal{L}(\boldsymbol{q}, \dot{\boldsymbol{q}}, \partial \boldsymbol{q} / \partial \boldsymbol{a}),
$$

with $L$ defined so that the action of (3.7) is given by $S=\int_{T} \mathrm{~d} t L$. Consequently, the canonical momentum is given by

$$
\boldsymbol{\Pi}=\frac{\delta L}{\delta \dot{\boldsymbol{q}}}=\rho_{0} \dot{\boldsymbol{q}}+\boldsymbol{\Pi}^{\star},
$$


and we see that we have a field theory counterpart to the finite-dimensional case for particle motion in a magnetic field where the kinetic momentum differs from the canonical momentum, here with the role of the vector potential being played by $\boldsymbol{\Pi}^{\star}$. Thus (6.1) gives the Hamiltonian

$$
H=\int_{D} \mathrm{~d}^{3} a\left(\frac{\left|\boldsymbol{\Pi}-\boldsymbol{\Pi}^{\star}\right|^{2}}{2 \rho_{0}}+\rho_{0} U\left(\frac{\rho_{0}}{\mathcal{J}}, \frac{\sigma_{0}}{\mathcal{J}}\right)+\frac{1}{2 \mathcal{J}} q_{, j}^{i} q_{, k}^{i} B_{0}^{j} B_{0}^{k}\right) .
$$

This Hamiltonian (6.4) together with the canonical Poisson bracket,

$$
\{F, G\}=\int_{D} \mathrm{~d}^{3} a\left(\frac{\partial F}{\partial \boldsymbol{q}} \cdot \frac{\partial G}{\partial \boldsymbol{\Pi}}-\frac{\partial G}{\partial \boldsymbol{q}} \cdot \frac{\partial F}{\partial \boldsymbol{\Pi}}\right),
$$

generates the Hamiltonian equations of motion in Lagrangian variables for our class of 3-D GVMHD models as follows:

$$
\dot{\boldsymbol{q}}=\{\boldsymbol{q}, H\}=\frac{\delta H}{\delta \boldsymbol{\Pi}} \quad \text { and } \quad \dot{\boldsymbol{\Pi}}=\{\boldsymbol{\Pi}, H\}=-\frac{\delta H}{\delta \boldsymbol{q}},
$$

equations equivalent to the Euler-Lagrange equations obtained via $\delta S=0$.

Now, one can use the Lagrange-Euler maps to convert both the Hamiltonian and the bracket into Eulerian variables. The procedure is described in the next section. We will see that the origin of the gyromap lies in (6.3) and how this expression relates to different choices of Eulerian variables. The bracket obtained in terms of any of these choices is endowed with Lie algebraic properties (Morrison 1998), most importantly the Jacobi identity, but it does not possess the canonical form of (6.5) because the Eulerian variables are not a set of canonical variables. As a result, one refers to the Hamiltonian and the bracket as being noncanonical in nature, and indeed one version is identical to that originally given in Morrison \& Greene (1980).

As the Lagrange-Euler maps are not one-to-one, the noncanonical brackets are degenerate in general, which gives rise to the existence of invariants - the Casimirs. The theory of Casimir invariants has been studied quite extensively (Morrison 1998, 2005; Holm 2008), but there are still unresolved subtleties regarding their incompleteness, see for example Yoshida, Morrison \& Dobarro (2014) and Yoshida \& Morrison (2014, 2016).

The Casimirs also possess several advantages of their own, such as variational principles for Eulerian equilibria of the form

$$
\delta F:=\delta(H+\lambda C)=0
$$

where $C$ represents any combination of all the known Casimirs. This procedure is known as the energy-Casimir method. Once the equilibria are known, the following symmetric operator can be constructed:

$$
\Lambda_{j k}:=\frac{\delta^{2} F}{\delta \psi^{j} \delta \psi^{k}},
$$

where $F$ is defined in (6.7) and the $\psi$ denote the Eulerian (noncanonical) variables. The energy-Casimir method states that the positive-definiteness of this operator is a sufficient condition for stability, although there are mathematical intricacies involved (Holm et al. 1985; Rein 1994; Batt, Morrison \& Rein 1995; Morrison 1998; Yoshida et al. 2003). Thus, the Eulerian noncanonical Hamiltonian description we obtain allows for implementation of such energy principles, although we will not pursue this application here. 


\subsection{The gyro-bracket}

We shall choose our new set of observables to be the Eulerian variables $\left\{\boldsymbol{M}^{c}, \rho, \sigma, \boldsymbol{B}\right\}$, where $\boldsymbol{M}^{c}$ was defined in (3.8). The reason for this choice will soon become obvious. Recall that the Lagrange-Euler maps can be expressed in an integral form, as they were for the density $\rho$ and canonical momentum density $\boldsymbol{M}^{c}$ in (2.6) and (2.7), respectively. The remaining Eulerian variables are given by

$$
\begin{aligned}
\sigma & =\int_{D} \mathrm{~d}^{3} a \delta(\boldsymbol{r}-\boldsymbol{q}) \sigma_{0}(\boldsymbol{a}), \\
B^{j} & =\int_{D} \mathrm{~d}^{3} a \delta(\boldsymbol{r}-\boldsymbol{q}) q_{, k}^{j} B_{0}^{k}(\boldsymbol{a}) .
\end{aligned}
$$

We use these expressions to obtain the noncanonical bracket from the canonical counterpart by the functional chain rule. Any functional of the Eulerian observables can be expressed in terms of $\boldsymbol{\Pi}$ and $\boldsymbol{q}$; hence to delineate, we denote functionals of $\boldsymbol{\Pi}$ and $\boldsymbol{q}$ by $\bar{F}$ and those in terms of the observables by $F$, and note symbolically that $\bar{F}=F \circ \mathfrak{E}$; consequently,

$$
\int_{D} \mathrm{~d}^{3} a\left[\frac{\delta \bar{F}}{\delta \boldsymbol{\Pi}} \cdot \delta \boldsymbol{\Pi}+\frac{\delta \bar{F}}{\delta \boldsymbol{q}} \cdot \delta \boldsymbol{q}\right]=\int_{D} \mathrm{~d}^{3} r\left[\frac{\delta F}{\delta \boldsymbol{M}^{c}} \cdot \delta \boldsymbol{M}^{c}+\frac{\delta F}{\delta \boldsymbol{B}} \cdot \delta \boldsymbol{B}+\frac{\delta F}{\delta \rho} \delta \rho+\frac{\delta F}{\delta \sigma} \delta \sigma\right] .
$$

From (2.6), we can conclude that

$$
\delta \rho=-\int_{D} \mathrm{~d}^{3} a \rho_{0} \nabla \delta(\boldsymbol{r}-\boldsymbol{q}) \cdot \delta \boldsymbol{q},
$$

and similar identities can be found for (2.6), (2.7), (6.9) and (6.10) as well. We substitute these identities into (6.11) and carry out integrations by parts, followed by a subsequent change in the order of integration. This results in terms that are dotted with $\delta \boldsymbol{q}$ and terms dotted with $\delta \boldsymbol{\Pi}$ on both the left- and right-hand sides of the expression. As $\delta \boldsymbol{q}$ and $\delta \boldsymbol{\Pi}$ are independent, these terms must balance and thereby we obtain relationships between the Eulerian and Lagrangian functional derivatives. The algebra involved is complicated, but quite straightforward, and we refer the reader to Morrison (2009) for a more pedagogical version. The final bracket that we obtain is found to be

$$
\begin{aligned}
\{F, G\}= & -\int \mathrm{d}^{3} r\left[M_{i}^{c}\left(\frac{\delta F}{\delta M_{j}^{c}} \partial_{j} \frac{\delta G}{\delta M_{i}^{c}}-\frac{\delta G}{\delta M_{j}^{c}} \partial_{j} \frac{\delta F}{\delta M_{i}^{c}}\right)\right. \\
& +\rho\left(\frac{\delta F}{\delta M_{j}^{c}} \partial_{j} \frac{\delta G}{\delta \rho}-\frac{\delta G}{\delta M_{j}^{c}} \partial_{j} \frac{\delta F}{\delta \rho}\right)+\sigma\left(\frac{\delta F}{\delta M_{j}^{c}} \partial_{j} \frac{\delta G}{\delta \sigma}-\frac{\delta G}{\delta M_{j}^{c}} \partial_{j} \frac{\delta F}{\delta \sigma}\right) \\
& \left.+B^{i}\left(\frac{\delta F}{\delta M_{j}^{c}} \partial_{j} \frac{\delta G}{\delta B^{i}}-\frac{\delta G}{\delta M_{j}^{c}} \partial_{j} \frac{\delta F}{\delta B^{i}}\right)+B^{i}\left(\frac{\delta G}{\delta B^{j}} \partial_{i} \frac{\delta F}{\delta M_{j}^{c}}-\frac{\delta F}{\delta B^{j}} \partial_{i} \frac{\delta G}{\delta M_{j}^{c}}\right)\right] .
\end{aligned}
$$

By inspection, one notices that the bracket derived above is exactly the same as the 3-D ideal MHD bracket (Morrison \& Greene 1980); however, here the canonical momentum $\boldsymbol{M}^{c}$ replaces the kinetic momentum $\boldsymbol{M}=\rho \boldsymbol{v}$.

Since the bracket (6.13) uses $\boldsymbol{M}^{c}$ as one of its observables, we must express our Hamiltonian in terms of this observable (and the others) as well. Because of the closure 
principle we know this is possible; indeed, (6.4) in Eulerian variables becomes

$$
H=\int \mathrm{d}^{3} r\left[\frac{\left|\boldsymbol{M}^{c}-\boldsymbol{M}^{\star}\right|^{2}}{2 \rho}+\rho U(\rho, \sigma,|\boldsymbol{B}|)+\frac{|\boldsymbol{B}|^{2}}{2}\right] .
$$

The Hamiltonian of (6.14) with the bracket of (6.13) generates our class of 3-D GVMHD models in the form

$$
\frac{\partial \mathfrak{E}}{\partial t}=\{\mathfrak{E}, H\} .
$$

On account of the fact that $\boldsymbol{M}=\boldsymbol{M}^{c}-\boldsymbol{M}^{\star}$, the energy has the form identical to that of ideal MHD. This is analogous to the fact that the kinetic energy for a charged particle in a magnetic field is identical to that for a free particle. Hence, there is a choice: one can either work with the standard ideal MHD bracket and the more complicated Hamiltonian of (6.14) in terms of the canonical momentum $\boldsymbol{M}^{c}$, or work with a complicated bracket written in terms of the variable $\boldsymbol{M}$, the conventional variable of magnetofluid theories, and the simpler ideal MHD Hamiltonian. To obtain the bracket in terms of $\boldsymbol{M}$ we can use the gyromap (3.8), $\boldsymbol{M}=\boldsymbol{M}^{c}-\boldsymbol{M}^{\star}$, in another chain rule calculation to transform from $\boldsymbol{M}^{c}$ to the variable $\boldsymbol{M}$. This is worked out in appendix B for the case $\boldsymbol{M}^{\star}=\nabla \times(\mathcal{F} \boldsymbol{B})$, giving rise to a complicated Poisson bracket.

Given that the noncanonical Poisson bracket in terms of $\boldsymbol{M}^{c}$ is the same as that of ideal MHD, it possesses the same Casimir invariants as the ideal MHD case if we replace $\boldsymbol{M}$ with $\boldsymbol{M}^{c}$. This use of the gyromap to obtain Casimirs, which first appeared in Morrison et al. (1984) and subsequently in other cases (Hazeltine et al. 1987; Izacard et al. 2011; Lingam \& Morrison 2014; Morrison et al. 2014), differs from most of the prior studies that have sought to derive Casimirs and other conserved invariants via the HAP approach using a variety of methods, see for example Morrison (1982, 1998), Padhye \& Morrison (1996a,b), Hameiri (2004) and Webb et al. $(2014 a, b)$ for a comprehensive discussion of the same.

So, for our present general gyroviscous models, the gyromap tells us that the $\boldsymbol{M}$-independent Casimirs of ideal MHD will be unchanged, an example being the magnetic helicity $\int \mathrm{d}^{3} \boldsymbol{r} \boldsymbol{A} \cdot \boldsymbol{B}$. On the other hand, the cross-helicity and other $\boldsymbol{M}$-dependent invariants are modified by the replacement $\boldsymbol{M} \rightarrow \boldsymbol{M}^{c}$. Thus, the new cross-helicity Casimir is given by

$$
\begin{aligned}
\int \mathrm{d}^{3} r \frac{\boldsymbol{M}^{c} \cdot \boldsymbol{B}}{\rho} & =\int \mathrm{d}^{3} r \frac{\left(\boldsymbol{M}+\boldsymbol{M}^{\star}\right) \cdot \boldsymbol{B}}{\rho} \\
& =\int \mathrm{d}^{3} r\left[\boldsymbol{v} \cdot \boldsymbol{B}+\left(\frac{\mathcal{F}}{\rho}\right) \boldsymbol{B} \cdot \boldsymbol{\nabla} \times \boldsymbol{B}\right] .
\end{aligned}
$$

Equation (6.16) is conserved for any choice of $\boldsymbol{M}^{\star}$ that satisfies the closure principle with a provision similar to that for conservation of the usual helicity of MHD, viz. that the flow be barotropic. In (6.17) we have inserted the special case of $\boldsymbol{M}^{\star}=\boldsymbol{\nabla} \times \boldsymbol{L}^{\star}$ with (3.10). The second term of (6.17) is proportional to the current helicity density, which is encountered regularly in the context of MHD (Moffatt 1978; Krause \& Raedler 1980; Brandenburg \& Subramanian 2005; Rincon 2019) and Hall MHD (Mininni, Gómez \& Mahajan 2003; Lingam \& Mahajan 2015; Lingam \& Bhattacharjee 2016a,b; Mahajan \& Lingam 2015, 2020) turbulence and dynamo theory. 


\section{Conclusions}

As we have noted in the introduction, there exist many approaches for constructing FLR models, each with their own advantages and disadvantages. In this paper, we present a HAP formalism that allows us to generate gyroviscous 3-D MHD models.

The action formalism allows us to clearly motivate and introduce the gyroviscous term, which is expressed in terms of a freely specifiable function. However, by using a combination of simple physical reasoning and prior results, we show that there exists a natural choice for this function, the 2-D limit of which exhibits consistency with the Braginskii gyroviscous tensor. We also show that the gyromap - a mathematical construct used to map back and forth between complicated Hamiltonians and easy brackets and vice versa - emerges naturally in this framework. The HAP formalism also has the distinct advantage of generating energy-conserving models from first principles, and all our models presented conserve both energy and momentum. Through the process of reduction, we recover the noncanonical bracket for this model, and a method for finding the Casimirs is elucidated.

One of the central results that emerged in this work was that the 3-D gyroviscous models do not conserve the orthodox angular momentum $\boldsymbol{r} \times \boldsymbol{M}$. We have presented a procedure for symmetrizing the momentum tensor via the construction of a hybrid momentum $\boldsymbol{M}^{\text {tot }}$. It is shown that the associated angular momentum $\boldsymbol{r} \times \boldsymbol{M}^{\text {tot }}$ is conserved. This procedure leads to the natural introduction of an intrinsic (spin) angular momentum which is likely to possess crucial ramifications in fusion and astrophysical plasmas; an example of the latter is briefly discussed.

The prospects for future work are manifold. The first, and perhaps the most important from a conceptual and mathematical standpoint, is to explore the putative violation of angular momentum conservation on a Lagrangian level. The second entails the application of this framework to astrophysical and fusion systems, and thereby assess whether the ensuing results are consistent with observations. The third involves a detailed comparison with other known gyroviscous tensors, such as those formulated by Braginskii (1965), Mikhailovskii \& Tsypin (1971), Liley (1972), Catto \& Simakov (2005), Ramos (2005a,b, 2010, 2011) and Simakov \& Molvig (2016). ${ }^{5}$ This is an ongoing effort, but preliminary results along this direction suggest that the symmetric part of our gyroviscous tensor might be compatible with results obtained by some of these authors, but at present we conclude that the 3-D version of Branginskii's gyroviscosity tensor probably does not emerge from an action principle. A comprehensive analysis is reserved for future publications. The comparison is more tedious (albeit feasible) for the full 3-D case in comparison with the 2-D case considered in Morrison et al. (1984), because the latter possessed a simple governing equation for the pressure, and it involved only two components of the momentum density and a single component of the magnetic field.

Our model was centred on the introduction of gyroviscosity into the ideal MHD model. However, given that several variants of extended MHD possess Lagrangian and Hamiltonian formulations (Keramidas Charidakos et al. 2014; Abdelhamid, Kawazura \& Yoshida 2015; Lingam, Morrison \& Miloshevich 2015a; Lingam, Morrison \& Tassi 2015b; D’Avignon, Morrison \& Lingam 2016; Lingam, Abdelhamid \& Hudson 2016a; Lingam, Miloshevich \& Morrison 2016b; Burby 2017; Miloshevich, Lingam \& Morrison 2017), it would seem natural to utilize the gyromap and thus formulate the gyroviscous contributions for this class of models; after doing so, their equilibria and stability can

\footnotetext{
${ }^{5}$ The stress tensor computed by Liley (1972) does not rely on the large- $B$ assumption, and reduces to the Braginskii gyroviscous tensor in the small-gyroradius limit (Hosking \& Marinoff 1973); see also Hosking \& Dewar (2016, § 2.8, 2.9).
} 
be obtained by using the HAP approach along the lines of Andreussi et al. (2010, 2012, 2013, 2016), Morrison et al. (2014) and Kaltsas, Throumoulopoulos \& Morrison (2017, 2018,2020 ) where the stability of a variety of equilibria is analysed using Lagrangian, energy-Casimir and dynamically accessibility methods. Likewise, this approach could also be extended to relativistic MHD and XMHD models with HAP formulations (D’Avignon, Morrison \& Pegoraro 2015; Grasso et al. 2017; Kawazura, Miloshevich \& Morrison 2017; Coquinot \& Morrison 2020; Ludwig 2020). We mention in passing that it would be interesting to explore how the time-dependent regauging of Andreussi et al. (2013) can be used to produce or remove the $\boldsymbol{M}^{\star}$-effects, in a manner analogous to how rotation can produce or remove effects of the magnetic field using Larmor's theorem.

Finally we mention a most basic extension of the present work. Our class of gyroviscous action principles were physically motivated, yet ultimately ad hoc. An alternative would be to start from a more basic model, such as the Vlasov-Maxwell system, and derive a gyroviscous action by asymptotic procedures. A natural starting point would be the Low Lagrangian (Low 1958) - see also Morrison \& Pfirsch (1989) and Morrison (2005) - and then reduce from phase space 'fluid' element variables of that theory to the usual fluid element that we have denoted here by $q(a, t)$. This would deviate from the usual historical approaches, which encompass most of the early literature, where one proceeds from ordering kinetic equations. Whichever route is taken, one typically uses intuition obtained from finite-dimensional particle orbit dynamics in given strong magnetic fields, and the associated drifts, in order to make approximations, often mixing up discrete particle orbit ideas with field theoretic perturbations. It was argued in Morrison, Vittot \& de Guillebon (2013) that a more consistent approach is to remain within the field theoretic framework, and it would appear prima facie that the Low Lagrangian is a natural framework for doing this. With this approach one could relate $\boldsymbol{M}^{\star}$ consistently to magnetization and other drifts on the fluid level. We hope to pursue this issue and others in the future.

\section{Acknowledgements}

P.J.M. received support from the US Department of Energy Contract no. DE-FG05-80ET-53088 during part of this work. A.W. thanks the Western New England University Research Fund for support. The authors thank the reviewers for their helpful feedback.

Editor Emanuele Tassi thanks the referees for their advice in evaluating this article.

\section{Declaration of interests}

The authors report no conflict of interest.

\section{Appendix A. An Euler-Poincaré approach to the 3-D gyromap}

The Lagrange-Euler maps, when expressed in an integral form, are given by (2.6) and (2.7). Instead of $\boldsymbol{M}^{c}$, we can also use the velocity as our observable, and it possesses the following Lagrange-Euler map:

$$
\boldsymbol{v}(\boldsymbol{r}, t)=\int_{D} \mathrm{~d}^{3} a \delta(\boldsymbol{r}-\boldsymbol{q}) \dot{\boldsymbol{q}}(\boldsymbol{a}, t) \mathcal{J},
$$

which is equivalent to $\boldsymbol{v}=\dot{\boldsymbol{q}}$, with the right-hand side evaluated at $\boldsymbol{a}=\boldsymbol{q}^{-1}(\boldsymbol{r}, t)$. The central idea is to express the Eulerian variations in terms of the Lagrangian ones, and thereby recover the equations of motion conveniently. The approach has classical roots, appeared in the plasma literature in the works of Frieman \& Rotenberg (1960), Katz (1961), 
Low (1961), Lundgren (1963), Calkin (1963), Merches (1969) and Newcomb (1962, 1972, 1973, 1983). The formalism was recast into geometric/group theoretic language in Holm et al. (1998), who gave it the title of the 'Euler-Poincaré'; this paper was motivated to a degree by what the authors called the 'Arnold program' (Arnold 1966). It should be pointed out that general variational principles of this form appeared in the early work of Hamel (1904). The method has subsequently been applied to very many systems, including kinetic theory (Cendra et al. 1998), complex fluids (Gay-Balmaz \& Ratiu 2009), reduced magnetofluid models (Brizard 2010b) and hybrid fluid-kinetic models (Holm \& Tronci 2012; Tronci \& Camporeale 2015; Burby \& Tronci 2017; Close, Burby \& Tronci 2018).

Let us illustrate this procedure by using the magnetic energy density as our example. We shall adopt the notation employed in Andreussi et al. (2013) for convenience, where the Lagrangian displacement $\delta \boldsymbol{q}$ is denoted by $\boldsymbol{\xi}$ and its Eulerianized counterpart is denoted by $\eta$. From (3.4), we know that

$$
\delta S_{\mathrm{mag}}=\int_{\mathcal{T}} \mathrm{d} t \int_{D} \mathrm{~d}^{3} r \boldsymbol{B} \cdot \delta \boldsymbol{B},
$$

where we have invoked the Eulerian closure principle. The final step lies in expressing $\delta \boldsymbol{B}$ in terms of $\boldsymbol{\eta}$, which has been undertaken in Frieman \& Rotenberg (1960) (see also Andreussi et al. 2013), which we list as follows:

$$
\delta \boldsymbol{B}=-\nabla \times(\boldsymbol{B} \times \boldsymbol{\eta}) .
$$

Upon using this in (A 2) and integrating by parts, we recover the $\boldsymbol{J} \times \boldsymbol{B}$ term, which is exactly the term arising in ideal MHD.

Upon applying the Euler-Poincaré method to (3.7), it can be verified that one does indeed recover (4.5) as our final result.

\section{Appendix B. The noncanonical gyroviscous bracket}

In (6.13), we presented the gyroviscous bracket in terms of the canonical momenta $M^{c}$ and the rest of the observables. The correspondence of the gyroviscous bracket with the ideal MHD bracket was also noted.

However, it is much more common to express noncanonical brackets in terms of the kinetic momentum $\boldsymbol{M}=\rho \boldsymbol{v}$, which we shall undertake here. In order to do so, we shall use the gyromap, discussed in $\S 3.2$,

$$
\boldsymbol{M}^{c}=\boldsymbol{M}+\boldsymbol{M}^{\star}=\boldsymbol{M}+\nabla \times(\mathcal{F} B),
$$

which can be easily rearranged to yield $\boldsymbol{M}=\boldsymbol{M}^{c}-\boldsymbol{M}^{\star}$. We shall now use the familiar concept that a given functional can be expressed in any set of (independent) observables. We denote by $F$ the functional in terms of $\boldsymbol{M}^{c}$ and the rest of the observables, and by $\tilde{F}$, the functional in terms of $\boldsymbol{M}$ and the rest. Since we know that $F \equiv \tilde{F}$, another chain rule calculation starts from

$$
\begin{aligned}
\delta F & =\int_{D} \mathrm{~d}^{3} r\left[\frac{\delta F}{\delta \boldsymbol{M}^{c}} \cdot \delta \boldsymbol{M}^{c}+\frac{\delta F}{\delta \boldsymbol{B}} \cdot \delta \boldsymbol{B}+\frac{\delta F}{\delta \rho} \delta \rho+\frac{\delta F}{\delta \sigma} \delta \sigma\right] \\
& =\int_{D} \mathrm{~d}^{3} r\left[\frac{\delta \tilde{F}}{\delta \boldsymbol{M}} \cdot \delta \boldsymbol{M}+\frac{\delta \tilde{F}}{\delta \boldsymbol{B}} \cdot \delta \boldsymbol{B}+\frac{\delta \tilde{F}}{\delta \rho} \delta \rho+\frac{\delta \tilde{F}}{\delta \sigma} \delta \sigma\right]=\delta \tilde{\boldsymbol{F}},
\end{aligned}
$$

and by using the gyromap, we find that

$$
\delta \boldsymbol{M}=\delta \boldsymbol{M}^{c}-\nabla \times[\delta(\mathcal{F} \boldsymbol{B})],
$$


and by substituting this into (B 2), integrating by parts and eliminating the resultant boundary terms, we finally recover the following relations:

$$
\left.\begin{array}{c}
\frac{\delta F}{\delta \boldsymbol{M}^{c}}=\frac{\delta \tilde{F}}{\delta \boldsymbol{M}}, \\
\frac{\delta F}{\delta \rho}=\frac{\delta \tilde{F}}{\delta \rho}-\boldsymbol{B} \cdot\left(\nabla \times \frac{\delta \tilde{F}}{\delta \boldsymbol{M}}\right) \frac{\partial \mathcal{F}}{\partial \rho}, \\
\frac{\delta F}{\delta \sigma}=\frac{\delta \tilde{F}}{\delta \sigma}-\boldsymbol{B} \cdot\left(\nabla \times \frac{\delta \tilde{F}}{\delta \boldsymbol{M}}\right) \frac{\partial \mathcal{F}}{\partial \sigma} \\
\frac{\delta F}{\delta \boldsymbol{B}}=\frac{\delta \tilde{F}}{\delta \boldsymbol{B}}-\left[\boldsymbol{B} \cdot\left(\nabla \times \frac{\delta \tilde{\boldsymbol{F}}}{\delta \boldsymbol{M}}\right)\right] \frac{\partial \mathcal{F}}{\partial|\boldsymbol{B}|} \frac{\boldsymbol{B}}{|\boldsymbol{B}|}-\left(\nabla \times \frac{\delta \tilde{\boldsymbol{F}}}{\delta \boldsymbol{M}}\right) \mathcal{F} .
\end{array}\right\}
$$

We can now recover the bracket in terms of $\boldsymbol{M}$ from (6.13), by implementing the following two successive steps.

(i) First, replace the $M_{i}^{c}$ in the first line of (6.13), prior to the functional derivatives, with (B 1). This ensures that only $\boldsymbol{M}$ and the other observables are present.

(ii) Next, the functional derivatives occurring in (6.13) should be replaced with the relations delineated in (B 4).

We shall not list the final bracket in its entirety since its complexity is clearly self-evident. ${ }^{6}$ Hence, this illustrates the advantage of the gyromap in facilitating a much simpler bracket. Simply through the process of inspection, it would have been almost impossible to construct the bracket in terms of $\boldsymbol{M}$ or to find the variable $\boldsymbol{M}^{c}$ that simplified the bracket.

The Hamiltonian, in terms of $\boldsymbol{M}$, is much simpler as seen from the following expression:

$$
H=\int \mathrm{d}^{3} r\left[\frac{|\boldsymbol{M}|^{2}}{2 \rho}+\rho U(\rho, \sigma)+\frac{|\boldsymbol{B}|^{2}}{2}\right] .
$$

In other words, the resultant Hamiltonian is exactly identical to the total energy associated with ideal MHD (Morrison \& Greene 1980; Freidberg 2014; Goedbloed et al. 2019).

We note, that any choice for $\boldsymbol{M}^{\star}$ that satisfies the Eulerian closure principle will, under an analogous transformation, yield a complicated bracket in terms of $\boldsymbol{M}$, yet one that reduces to the MHD bracket of Morrison \& Greene (1980) when the variable $\boldsymbol{M}^{c}$ is used. Thus, for any choice we have the same trade-off between Hamiltonian and bracket.

\section{REFERENCES}

Abdelhamid, H. M., Kawazura, Y. \& Yoshida, Z. 2015 Hamiltonian formalism of extended magnetohydrodynamics. J. Phys. A: Math. Gen. 48 (23), 235502.

Andreussi, T., Morrison, P. J. \& Pegoraro, F. 2010 MHD equilibrium variational principles with symmetry. Plasma Phys. Control. Fusion 52 (5), 055001.

Andreussi, T., Morrison, P. J. \& PEgORARO, F. 2012 Hamiltonian magnetohydrodynamics: helically symmetric formulation, Casimir invariants, and equilibrium variational principles. Phys. Plasmas 19 (5), 052102.

\footnotetext{
${ }^{6}$ An explicit example of the gyro-bracket in terms of $\boldsymbol{M}$ for a particular choice of the gyromap was presented in A. Wurm and P. J. Morrison, Derivation of Hamiltonian magnetofluid models with gyroviscous-like contributions using a gyromap, Sherwood International Fusion Theory conference, Santa Fe, NM, April 15-17 (2013).
} 
Andreussi, T., Morrison, P. J. \& Pegoraro, F. 2013 Hamiltonian magnetohydrodynamics: Lagrangian, Eulerian, and dynamically accessible stability-theory. Phys. Plasmas 20 (9), 092104.

Andreussi, T., Morrison, P. J. \& Pegoraro, F. 2016 Hamiltonian magnetohydrodynamics: Lagrangian, Eulerian, and dynamically accessible stability-examples with translation symmetry. Phys. Plasmas 23 (10), 102112.

ARNOLD, V. I. 1966 Sur la géométrie différentielle des groupes de Lie de dimension infinie et ses applications à l'hydrodynamique des fluides parfaits. Ann. Inst. Fourier Grenoble 16 (1), 319-361.

ARNolD, V. I. 1978 Mathematical Methods of Classical Mechanics. Springer.

ARnOlD, V. I. \& KHESIN, B. A. 1998 Topological methods in hydrodynamics, Applied Mathematical Sciences, vol. 125. Springer.

BAtT, J., Morrison, P. J. \& REIN, G. 1995 Linear stability of stationary solutions of the Vlasov-Poisson system in three dimensions. Arch. Rat. Mech. Anal. 130 (2), 163-182.

Beer, M. A. \& Hammett, G. W. 1996 Toroidal gyrofluid equations for simulations of tokamak turbulence. Phys. Plasmas 3 (11), 4046-4064.

Belova, E. V. 2001 Nonlinear gyroviscous force in a collisionless plasma. Phys. Plasmas 8 (9), 3936-3944.

Bennett, A. 2006 Lagrangian Fluid Dynamics. Cambridge University Press.

BILlig, Y. 2005 Magnetic hydrodynamics with asymmetric stress tensor. J. Math. Phys. 46 (4), 043101.

Bodenheimer, P. 1995 Angular momentum evolution of young stars and disks. Annu. Rev. Astron. Astrophys. 33, 199-238.

Bouvier, J., Matt, S. P., Mohanty, S., Scholz, A., Stassun, K. G. \& Zanni, C. 2014 Angular momentum evolution of young low-mass stars and brown dwarfs: observations and theory. In Protostars and Planets VI (ed. H. Beuther, R. S. Klessen, C. P. Dullemond \& T. Henning), pp. 433-450.

BOWERS, E. 1971 Finite-Larmor-radius equations for coffisionless plasmas in general magnetic fields. J. Plasma Phys. 6 (1), 87-105.

BRAGINSKII, S. I. 1958 Transport phenomena in a completely ionized two-temperature plasma. Sov. J. Exp. Theor. Phys. 6, 358-369.

BRAgINSKII, S. I. 1965 Transport processes in a plasma. Rev. Plasma Phys. 1, 205-311.

Brandenburg, A. \& Subramanian, K. 2005 Astrophysical magnetic fields and nonlinear dynamo theory. Phys. Rep. 417 (1-4), 1-209.

BRIZARD, A. 1992 Nonlinear gyrofluid description of turbulent magnetized plasmas. Phys. Fluids B 4 (5), $1213-1228$.

BRIZARD, A. J. $2010 a$ Classical Zitterbewegung in reduced plasma dynamics. arXiv:1010.5710.

BRIZARD, A. J. $2010 b$ Noether derivation of exact conservation laws for dissipationless reduced-fluid models. Phys. Plasmas 17 (11), 112503

Brizard, A. J., Denton, R. E., Rogers, B. \& Lotko, W. 2008 Nonlinear finite-Larmor-radius effects in reduced fluid models. Phys. Plasmas 15 (8), 082302.

Burby, J. W. 2017 Magnetohydrodynamic motion of a two-fluid plasma. Phys. Plasmas 24 (8), 082104.

Burby, J. W. \& TRONCI, C. 2017 Variational approach to low-frequency kinetic-MHD in the current coupling scheme. Plasma Phys. Control. Fusion 59 (4), 045013.

CALKin, M. G. 1963 An action principle for magnetohydrodynamics. Can. J. Phys. 41, 2241.

Callen, J. D., Qu, W. X., Siebert, K. D., Carreras, B. A., Shaing, K. C. \& Spong, D. A. 1987 Neoclassical MHD equations, instabilities and transport in tokamaks. In Plasma Physics and Controlled Nuclear Fusion Research 1986, vol. 2, pp. 157-166. IAEA.

CAтto, P. J. \& Simakov, A. N. 2005 A new, explicitly collisional contribution to the gyroviscosity and the radial electric field in a collisional tokamak. Phys. Plasmas 12 (11), 114503.

Cendra, H., Holm, D. D., Hoyle, M. J. W. \& Marsden, J. E. 1998 The Maxwell-Vlasov equations in Euler-Poincaré form. J. Math. Phys. 39 (6), 3138-3157.

Chapman, S. \& Cowling, T. G. 1970 The Mathematical Theory of Non-Uniform Gases: An Account of the Kinetic Theory of Viscosity, Thermal Conduction and Diffusion in Gases, 3rd edn. Cambridge University Press.

Chew, G. F., Goldberger, M. L. \& Low, F. E. 1956 The Boltzmann Equation and the one-fluid hydromagnetic equations in the absence of particle collisions. Proc. R. Soc. Lond. A 236 (1204), $112-118$. 
Clebsch, R. F. A. 1857 Uber eine allgemeine Transformation der hydrodynamischen Gleichungen. J. Reine Angew. Math. 54, 293-312.

Clebsch, R. F. A. 1859 Ueber die Integration der hydrodynamischen Gleichungen. J. Reine Angew. Math. $56,1-10$.

Close, A. R. D., Burby, J. W. \& Tronci, C. 2018 A low-frequency variational model for energetic particle effects in the pressure-coupling scheme. J. Plasma Phys. 84 (4), 905840401.

Comisso, L., Grasso, D., Waelbroeck, F. L. \& Borgogno, D. 2013 Gyro-induced acceleration of magnetic reconnection. Phys. Plasmas 20 (9), 092118.

Coquinot, B. \& Morrison, P. J. 2020 A general metriplectic framework with application to dissipative extended magnetohydrodynamics. J. Plasma Phys. 86 (3), 835860302.

DÁvignon, E., Morrison, P. J. \& Pegoraro, F. 2015 Action principle for relativistic magnetohydrodynamics. Phys. Rev. D 91 (8), 084050.

Dvignon, E. C., Morrison, P. J. \& Lingam, M. 2016 Derivation of the Hall and extended magnetohydrodynamics brackets. Phys. Plasmas 23 (6), 062101.

Dewar, R. L. 1977 Energy-momentum tensors for dispersive electromagnetic waves. Aust. J. Phys. 30, 533.

Diamond, P. H., Kosuga, Y., Gürcan, Ö. D., McDevitt, C. J., Hahm, T. S., Fedorczak, N., Rice, J. E., WANG, W. X., KU, S., KWON, J. M., et al. 2013 An overview of intrinsic torque and momentum transport bifurcations in toroidal plasmas. Nucl. Fusion 53 (10), 104019.

Evans, D. J. 1979 The nonsymmetric pressure tensor in polyatomic fluids. J. Stat. Phys. 20 (5), 547-555.

FREIDBERG, J. P. 2014 Ideal MHD. Cambridge University Press.

Frieman, E. \& Rotenberg, M. 1960 On hydromagnetic stability of stationary equilibria. Rev. Mod. Phys. 32 (4), 898-902.

Frisch, U., Grimberg, G. \& Villone, B. 2017 A contemporary look at Hermann Hankel's 1861 pioneering work on Lagrangian fluid dynamics. Eur. Phys. J. H 42 (4), 537-556. DOI:10.1140/epjh/e2017-80038-2.

FRISCH, U. \& VILLONE, B. 2014 Cauchy's almost forgotten Lagrangian formulation of the Euler equation for 3D incompressible flow. Eur. Phys. J. H 39 (3), 325-351.

Gay-Balmaz, F. \& Ratiu, T. S. 2009 The geometric structure of complex fluids. Adv. Appl. Maths 42 (2), 176-275.

DE Gennes, P.-G. \& PRost, J. 1993 The physics of liquid crystals. In International Series of Monographs on Physics, 2nd edn., vol. 83. Oxford University Press.

Goedbloed, H., Keppens, R. \& Poedts, S. 2019 Magnetohydrodynamics of Laboratory and Astrophysical Plasmas. Cambridge University Press.

Goswami, P., Passot, T. \& Sulem, P. L. 2005 A Landau fluid model for warm collisionless plasmas. Phys. Plasmas 12 (10), 102109.

DE Grassie, J. S. 2009 Tokamak rotation sources, transport and sinks. Plasma Phys. Control. Fusion 51 (12), 124047.

Grasso, D., TAssi, E., Abdelhamid, H. M. \& Morrison, P. J. 2017 Structure and computation of two-dimensional incompressible extended MHD. Phys. Plasmas 24 (1), 012110.

GÜRCAN, Ö. D., DiAmond, P. H., HAhM, T. S. \& Singh, R. 2007 Intrinsic rotation and electric field shear. Phys. Plasmas 14 (4), 042306.

HAmeiri, E. 2004 The complete set of Casimir constants of the motion in magnetohydrodynamics. Phys. Plasmas 11 (7), 3423-3431.

Hamel, G. 1904 Die Lagrange-Eulerschen gleichungen der mechanik. Zeitschr. für Math. u. Phys. 50, $1-57$.

Hammett, G. W., Dorland, W. \& Perkins, F. W. 1992 Fluid models of phase mixing, Landau damping, and nonlinear gyrokinetic dynamics. Phys. Fluids B 4 (7), 2052-2061.

HANKE, H. 1861 Zur allgemeinen Theorie der Bewegung der Flussigkeiten. Dieterichschen Univ.-Buchdruckerei.

Hartmann, L., Herczeg, G. \& Calvet, N. 2016 Accretion onto pre-main-sequence stars. Annu. Rev. Astron. Astrophys. 54, 135-180. 
Hasegawa, A. \& WaKatAni, M. 1983 Finite-Larmor-radius magnetohydrodynamic equations for microturbulence. Phys. Fluids 26 (10), 2770-2772.

Hazeltine, R. D., Hsu, C. T. \& Morrison, P. J. 1987 Hamiltonian four-field model for nonlinear tokamak dynamics. Phys. Fluids 30 (10), 3204-3211.

Hazeltine, R. D., Mahajan, S. M. \& Morrison, P. J. 2013 Local thermodynamics of a magnetized, anisotropic plasma. Phys. Plasmas 20 (2), 022506.

Hazeltine, R. D. \& Meiss, J. D. 1992 Plasma Confinement. Addison-Wesley.

Hazeltine, R. D. \& Waelbroeck, F. L. 1998 The Framework of Plasma Physics. Perseus Books.

von Helmholtz, H. 1858 Uber Integrale der hydrodynamischen Gleichungen, welche den Wirbelbewegungen entsprechen. J. Reine Angew. Math. 55, 25-55.

Holm, D. D. 2008 Geometric Mechanics: Dynamics and symmetry. Imperial College Press.

Holm, D. D., Marsden, J. E. \& RatiU, T. 1998 The Euler-Poincaré equations and semidirect products with applications to continuum theories. Adv. Maths 137, 1-81.

Holm, D. D., Marsden, J. E., Ratiu, T. \& Weinstein, A. 1985 Nonlinear stability of fluid and plasma equilibria. Phys. Rep. 123 (1-2), 1-116.

Holm, D. D. \& Tronci, C. 2012 Euler-Poincaré formulation of hybrid plasma models. Commun. Math. Sci. 10 (1), 191-222.

Hosking, R. J. \& Dewar, R. L. 2016 Fundamental Fluid Mechanics and Magnetohydrodynamics. Springer.

Hosking, R. J. \& MARINOFF, G. M. 1973 Magneto-viscous effects on the ideal and resistive gravitational instabilities in Cartesian geometry. Plasma Phys. 15 (5), 327-341.

Hsu, C. T., Hazeltine, R. D. \& Morrison, P. J. 1986 A generalized reduced fluid model with finite ion-gyroradius effects. Phys. Fluids 29 (5), 1480-1487.

Izacard, O., Chandre, C., TAssi, E. \& Ciraolo, G. 2011 Gyromap for a two-dimensional Hamiltonian fluid model derived from Braginskii's closure for magnetized plasmas. Phys. Plasmas 18 (6), 062105.

JACKSON, J. D. 1998 Classical Electrodynamics, 3rd edn. John Wiley \& Sons.

Kaltsas, D. A., Throumoulopoulos, G. N. \& Morrison, P. J. 2017 Translationally symmetric extended MHD via Hamiltonian reduction: energy-Casimir equilibria. Phys. Plasmas 24 (9), 092504.

Kaltsas, D. A., Throumoulopoulos, G. N. \& Morrison, P. J. 2018 Helically symmetric extended magnetohydrodynamics: Hamiltonian formulation and equilibrium variational principles. J. Plasma Phys. 84 (3), 745840301.

Kaltsas, D. A., Throumoulopoulos, G. N. \& Morrison, P. J. 2020 Energy-Casimir, dynamically accessible, and Lagrangian stability of extended magnetohydrodynamic equilibria. Phys. Plasmas 27 (1), 012104.

KATZ, S. 1961 Lagrangean density for an inviscid, perfect, compressible plasma. Phys. Fluids 4 (3), 345-348.

Kaufman, A. N. 1960 Plasma viscosity in a magnetic field. Phys. Fluids 3 (4), 610-616.

Kawazura, Y., Miloshevich, G. \& Morrison, P. J. 2017 Action principles for relativistic extended magnetohydrodynamics: a unified theory of magnetofluid models. Phys. Plasmas 24 (2), 022103.

Kennel, C. F. \& Greene, J. M. 1966 Finite Larmor radius hydromagnetics. Ann. Phys. 38 (1), 63-94.

Keramidas Charidakos, I., Lingam, M., Morrison, P. J., White, R. L. \& Wurm, A. 2014 Action principles for extended magnetohydrodynamic models. Phys. Plasmas 21 (9), 092118.

Keramidas Charidakos, I., Waelbroeck, F. L. \& Morrison, P. J. 2015 A Hamiltonian five-field gyrofluid model. Phys. Plasmas 22 (11), 112113.

KIMURA, K. \& MORRISON, P. J. 2014 On energy conservation in extended magnetohydrodynamics. Phys. Plasmas 21 (8), 082101.

KIRCHHOFF, R. 1876 Vorlesungenuber Mathematische Physik: Mechanik. Teubner.

KOPCZYŃSKI, W. 1990 Variational principles for gravity and fluids. Ann. Phys. 203 (2), 308-338.

Krause, F. \& RAedler, K. H. 1980 Mean-Field Magnetohydrodynamics and Dynamo Theory. Pergamon.

Krommes, J. A. \& KolesniKov, R. A. 2004 Hamiltonian description of convective-cell generation. Phys. Plasmas 11 (6), L29-L32. 
Kuvshinov, B. N., Pegoraro, F. \& Schep, T. J. 1994 Hamiltonian formulation of low-frequency, nonlinear plasma dynamics. Phys. Lett. A 191 (3-4), 296-300.

Lagrange, J. L. 1789 Mécanique Analytique. Mme. Ve. Courcier.

LiLEY, B. S. 1972 Transport equations for a plasma. Unpublished report Phys. No. 103. University of Waikato.

LingAM, M. 2015a Dissipative effects in magnetohydrodynamical models with intrinsic magnetization. Commun. Nonlinear Sci. Numer. Simul. 28 (1-3), 223-231.

Lingam, M. $2015 b$ Hall viscosity: a link between quantum Hall systems, plasmas and liquid crystals. Phys. Lett. A 379 (22-23), 1425-1430.

LingAM, M. 2015c Hamiltonian and action principle formalisms for spin-1/2 magnetohydrodynamics. Phys. Plasmas 22 (2), 022124.

LingAM, M. 2015d Hamiltonian and action principle formulations of plasma fluid models. PhD thesis, The University of Texas at Austin.

Lingam, M., Abdelhamid, H. M. \& Hudson, S. R. $2016 a$ Multi-region relaxed Hall magnetohydrodynamics with flow. Phys. Plasmas 23 (8), 082103.

Lingam, M. \& BhatTACHARJEe, A. 2016a A heuristic model for MRI turbulent stresses in Hall MHD. Mon. Not. R. Astron. Soc. 460 (1), 478-488.

Lingam, M. \& BhattacharJee, A. $2016 b$ Hall current effects in mean-field dynamo theory. Astrophys. J. 829 (1), 51.

Lingam, M. \& LoEB, A. 2018 Physical constraints on the likelihood of life on exoplanets. Intl J. Astrobiol. 17 (2), 116-126.

Lingam, M. \& LoEB, A. 2019 Colloquium: physical constraints for the evolution of life on exoplanets. Rev. Mod. Phys. 91 (2), 021002.

LingaM, M. \& MAHAJAN, S. M. 2015 Modelling astrophysical outflows via the unified dynamo-reverse dynamo mechanism. Mon. Not. R. Astron. Soc. 449, L36-L40.

Lingam, M., Miloshevich, G. \& Morrison, P. J. 2016b Concomitant Hamiltonian and topological structures of extended magnetohydrodynamics. Phys. Lett. A 380 (31-32), 2400-2406.

LingaM, M. \& MORRISON, P. J. 2014 The action principle for generalized fluid motion including gyroviscosity. Phys. Lett. A 378 (47), 3526-3532.

Lingam, M., Morrison, P. J. \& Miloshevich, G. 2015a Remarkable connections between extended magnetohydrodynamics models. Phys. Plasmas 22 (7), 072111.

Lingam, M., Morrison, P. J. \& TASsi, E. $2015 b$ Inertial magnetohydrodynamics. Phys. Lett. A 379 (6), 570-576.

Low, F. E. 1958 A Lagrangian formulation of the Boltzmann-Vlasov equation for plasmas. Proc. R. Soc. Lond. A 248 (1253), 282-287.

Low, F. E. 1961 Persistence of stability in Lagrangian systems. Phys. Fluids 4 (7), 842-846.

Ludwig, G. O. 2020 Variational formulation of plasma dynamics. Phys. Plasmas 27 (2), 022110.

LUNDGREN, T. S. 1963 Hamilton's variational principle for a perfectly conducting plasma continuum. Phys. Fluids 6 (7), 898-904.

MACMAHON, A. 1965 Finite gyro-radius corrections to the hydromagnetic equations for a Vlasov plasma. Phys. Fluids 8 (10), 1840-1845.

Madsen, J. 2013 Full-F gyrofluid model. Phys. Plasmas 20 (7), 072301.

Mahajan, S. M. \& LingaM, M. 2015 Multi-fluid systems-Multi-Beltrami relaxed states and their implications. Phys. Plasmas 22 (9), 092123.

Mahajan, S. M. \& Lingam, M. 2020 Constraining Alfvénic turbulence with helicity invariants. Mon. Not. R. Astron. Soc. 495 (3), 2771-2776.

MATT, S. \& PUdRITZ, R. E. 2005 Accretion-powered stellar winds as a solution to the stellar angular momentum problem. Astrophys. J. Lett. 632 (2), L135-L138.

MCLennan, J. A. 1966 Symmetry of the stress tensor. Physica 32 (4), 689-692.

Merches, I. 1969 Variational principle in magnetohydrodynamics. Phys. Fluids 12 (10), 2225-2227.

MiKhaIlovs KII, A. B. 1992 Electromagnetic Instabilities in an Inhomogeneous Plasma. IOP Publishing.

Mikhailovskit, A. B. \& TsYPIN, V. S. 1971 Transport equations and gradient instabilities in a high pressure collisional plasma. Plasma Phys. 13 (9), 785-798. 
Miloshevich, G., Lingam, M. \& Morrison, P. J. 2017 On the structure and statistical theory of turbulence of extended magnetohydrodynamics. New J. Phys. 19 (1), 015007.

Mininni, P. D., Gómez, D. O. \& Mahajan, S. M. 2003 Dynamo action in magnetohydrodynamics and Hall-magnetohydrodynamics. Astrophys. J. 587 (1), 472-481.

Moffatt, H. K. 1978 Magnetic Field Generation in Electrically Conducting Fluids. Cambridge University Press.

MORRISON, P. J. 1982 Poisson brackets for fluids and plasmas. In American Institute of Physics Conference Series (ed. M. Tabor \& Y. M. Treve), vol. 88, pp. 13-46. American Institute of Physics Conference Series.

Morrison, P. J. 1998 Hamiltonian description of the ideal fluid. Rev. Mod. Phys. 70 (2), 467-521.

MorRison, P. J. 2005 Hamiltonian and action principle formulations of plasma physicsa. Phys. Plasmas $12(5), 058102$.

MorRison, P. J. 2009 On Hamiltonian and action principle formulations of plasma dynamics. In American Institute of Physics Conference Series (ed. B. Eliasson \& P. K. Shukla), vol. 1188, pp. 329-344. American Institute of Physics Conference Series.

Morrison, P. J. 2017 Structure and structure-preserving algorithms for plasma physics. Phys. Plasmas 24 (5), 055502.

Morrison, P. J., Caldas, I. L. \& TAsso, H. 1984 Hamiltonian formulation of two-dimensional Gyroviscous MHD. Z. Naturforsch. Teil A 39 (11), 1023-1027.

Morrison, P. J. \& GREENE, J. M. 1980 Noncanonical Hamiltonian density formulation of hydrodynamics and ideal magnetohydrodynamics. Phys. Rev. Lett. 45 (10), 790-794.

MoRrison, P. J. \& HAZELTINE, R. D. 1984 Hamiltonian formulation of reduced magnetohydrodynamics. Phys. Fluids 27 (4), 886-897.

Morrison, P. J., Lingam, M. \& Acevedo, R. 2014 Hamiltonian and action formalisms for two-dimensional gyroviscous magnetohydrodynamics. Phys. Plasmas 21 (8), 082102.

Morrison, P. J. \& PFIRSCH, D. 1989 Free-energy expressions for Vlasov equilibria. Phys. Rev. A 40 (7), 3898-3910.

Morrison, P. J., Vittot, M. \& De Guillebon, L. 2013 Lifting particle coordinate changes of magnetic moment type to Vlasov-Maxwell Hamiltonian dynamics. Phys. Plasmas 20 (3), 032109.

NewCOMB, W. A. 1962 Lagrangian and Hamiltonian methods in magnetohydrodynamics. Nucl. Fusion Suppl. pt 2, 451-463.

NewCOMB, W. A. 1972 The perfect gyroscopic fluid. Ann. Phys. 72 (1), 29-79.

Newcomb, W. A. 1973 Gyroscopic-quasielastic fluid systems. Ann. Phys. 81 (1), 231-331.

Newcomb, W. A. 1983 The anisorrhopic guiding-center fluid. Ann. Phys. 150 (1), 172-266.

Olmsted, R. D. \& SNider, R. F. 1976 Symmetry of the pressure tensor in macromolecular fluids. J. Chem. Phys. 65 (9), 3423-3426.

PAdhye, N. \& Morrison, P. J. $1996 a$ Fluid element relabeling symmetry. Phys. Lett. A 219 (5-6), 287-292.

PAdhye, N. \& Morrison, P. J. $1996 b$ Relabeling symmetries in hydrodynamics and magnetohydrodynamics. Plasma Phys. Rep. 22 (10), 869-877.

Papapetrou, A. 1949 LXXXVII. Non-symmetric stress-energy-momentum tensor and spin-density. Lond. Edin. Dublin Phil. Mag. J. Sci. 40 (308), 937-946.

PAssot, T. \& Sulem, P. L. 2007 Collisionless magnetohydrodynamics with gyrokinetic effects. Phys. Plasmas 14 (8), 082502.

Passot, T., Sulem, P. L. \& Hunana, P. 2012 Extending magnetohydrodynamics to the slow dynamics of collisionless plasmas. Phys. Plasmas 19 (8), 082113.

PASsot, T., SUlEM, P. L. \& TASSI, E. 2017 Electron-scale reduced fluid models with gyroviscous effects. J. Plasma Phys. 83 (4), 715830402.

Passot, T., Sulem, P. L. \& TAssi, E. 2018 Gyrofluid modeling and phenomenology of low- $\beta_{e}$ Alfvén wave turbulence. Phys. Plasmas 25 (4), 042107.

Pfefferlé, D., HirvijoKi, E. \& Lingam, M. 2017 Exact collisional moments for plasma fluid theories. Phys. Plasmas 24 (4), 042118.

PFIRSCH, D. \& MORRISON, P. J. 1985 Local conservation laws for the Maxwell-Vlasov and collisionless kinetic guiding-center theories. Phys. Rev. A 32 (3), 1714-1721. 
Pogutse, I. O., Smolyakov, A. I. \& Hirose, A. 1998 Magnetohydrodynamic equations for plasmas with finite-Larmor-radius effects. J. Plasma Phys. 60 (1), 133-149.

PRIEST, E. 2014 Magnetohydrodynamics of the Sun. Cambridge University Press.

Ramos, J. J. 2005 a Fluid formalism for collisionless magnetized plasmas. Phys. Plasmas 12 (5), 052102.

RAmos, J. J. $2005 b$ General expression of the gyroviscous force. Phys. Plasmas 12 (11), 112301.

RAmos, J. J. 2007 Fluid theory of magnetized plasma dynamics at low collisionality. Phys. Plasmas $14(5), 052506$.

Ramos, J. J. 2010 Fluid and drift-kinetic description of a magnetized plasma with low collisionality and slow dynamics orderings. I. Electron theory. Phys. Plasmas 17 (8), 082502.

Ramos, J. J. 2011 Fluid and drift-kinetic description of a magnetized plasma with low collisionality and slow dynamics orderings. II. Ion theory. Phys. Plasmas 18 (10), 102506.

REIN, G. 1994 Non-linear stability for the Vlasov-Poisson system-the energy-Casimir method. Math. Meth. Appl. Sci. 17 (14), 1129-1140.

RICE, J. E. 2016 Experimental observations of driven and intrinsic rotation in tokamak plasmas. Plasma Phys. Control. Fusion 58 (8), 083001.

RinCon, F. 2019 Dynamo theories. J. Plasma Phys. 85 (4), 205850401.

Roberts, K. V. \& TAYLOR, J. B. 1962 Magnetohydrodynamic equations for finite larmor radius. Phys. Rev. Lett. 8 (5), 197-198.

Rosenbluth, M. N. \& Simon, A. 1965 Finite Larmor radius equations with nonuniform electric fields and velocities. Phys. Fluids 8 (7), 1300-1322.

Rosensweig, R. E. 1985 Ferrohydrodynamics. Cambridge University Press.

Scholz, A. 2009 Stellar spindown: from the ONC to the sun. In 15th Cambridge Workshop on Cool Stars, Stellar Systems, and the Sun (ed. E. Stempels), vol. 1094, pp. 61-70. American Institute of Physics Conference Series.

ScotT, B. \& SMIRnOV, J. 2010 Energetic consistency and momentum conservation in the gyrokinetic description of tokamak plasmas. Phys. Plasmas 17 (11), 112302.

ScotT, B. D. 2005 Free-energy conservation in local gyrofluid models. Phys. Plasmas 12 (10), 102307.

SCOTT, B. D. 2007 Nonlinear polarization and dissipative correspondence between low-frequency fluid and gyrofluid equations. Phys. Plasmas 14 (10), 102318.

Seliger, R. L. \& Whitham, G. B. 1968 Variational principles in continuum mechanics. Proc. R. Soc. Lond. A 305 (1480), 1-25.

SerRIN, J. 1959 Mathematical principles of classical fluid mechanics. In Handbuch der Physik (ed. S. Flügge), vol. VIII, pp. 125-263. Springer.

Simakov, A. N. \& CATTO, P. J. 2006 What are the limitations of Braginskii's fluid equations and Hazeltine's drift kinetic equation? In Theory of Fusion Plasmas (ed. O. Sauter), vol. 871, pp. 238-249. American Institute of Physics Conference Series.

SimaKOV, A. N. \& MOLVIG, K. 2016 Hydrodynamic description of an unmagnetized plasma with multiple ion species. I. General formulation. Phys. Plasmas 23 (3), 032115.

Similon, P. L. 1985 Conservation laws for relativistic guiding-center plasma. Phys. Lett. A 112 (1-2), 33-37.

Smolyakov, A. I., Pogutse, I. O. \& Hirose, A. 1995 Fluid model of collisionless plasma with finite Larmor radius effects. Phys. Plasmas 2 (12), 4451-4454.

SNIDER, R. F. \& LEWCHUK, K. S. 1967 Irreversible thermodynamics of a fluid system with spin. J. Chem. Phys. 46 (8), 3163-3172.

SNYDER, P. B. \& HAMmetT, G. W. 2001 A Landau fluid model for electromagnetic plasma microturbulence. Phys. Plasmas 8 (7), 3199-3216.

Snyder, P. B., Hammett, G. W. \& Dorland, W. 1997 Landau fluid models of collisionless magnetohydrodynamics. Phys. Plasmas 4 (11), 3974-3985.

Staebler, G. M., Kinsey, J. E. \& Waltz, R. E. 2005 Gyro-Landau fluid equations for trapped and passing particles. Phys. Plasmas 12 (10), 102508.

Sudarshan, E. C. G. \& Mukunda, N. 2016 Classical Dynamics: A Modern Perspective, 2nd edn. World Scientific.

Sulem, P. L. \& PASsot, T. 2008 FLR Landau fluids for collisionless plasmas. Commun. Nonlinear Sci. Numer. Simul. 13 (1), 189-196. 
TASSI, E. 2017 Hamiltonian closures in fluid models for plasmas. Eur. Phys. J. D 71 (11), 269.

TASSI, E. 2019 Hamiltonian gyrofluid reductions of gyrokinetic equations. J. Phys. A: Math. Gen. 52 (46), 465501.

Tassi, E., Grasso, D., Borgogno, D., Passot, T. \& Sulem, P. L. 2018 A reduced Landau-Gyrofluid model for magnetic reconnection driven by electron inertia. J. Plasma Phys. 84 (4), 725840401.

Tassi, E., Grasso, D. \& Pegoraro, F. $2010 a$ Nonlinear processes in Hamiltonian reconnection. Commun. Nonlinear Sci. Numer. Simul. 15 (1), 2-9.

Tassi, E., Morrison, P. J., Grasso, D. \& Pegoraro, F. $2010 b$ Hamiltonian four-field model for magnetic reconnection: nonlinear dynamics and extension to three dimensions with externally applied fields. Nucl. Fusion 50 (3), 034007.

Tassi, E., Morrison, P. J., Waelbroeck, F. L. \& Grasso, D. 2008 Hamiltonian formulation and analysis of a collisionless fluid reconnection model. Plasma Phys. Control. Fusion 50 (8), 085014.

Tronci, C. \& CAmporeale, E. 2015 Neutral Vlasov kinetic theory of magnetized plasmas. Phys. Plasmas 22 (2), 020704.

Tronci, C., Tassi, E., Camporeale, E. \& Morrison, P. J. 2014 Hybrid Vlasov-MHD models: Hamiltonian vs. non-Hamiltonian. Plasma Phys. Control. Fusion 56 (9), 095008.

Truesdell, C. \& Toupin, R. 1960 The Classical Field Theories. Handbuch der Physik 2, 226-858.

TUR, A. V. \& YANOVSKY, V. V. 1993 Invariants in dissipationless hydrodynamic media. J. Fluid Mech. 248, 67-106.

Waelbroeck, F. L., Hazeltine, R. D. \& Morrison, P. J. 2009 A Hamiltonian electromagnetic gyrofluid model. Phys. Plasmas 16 (3), 032109.

WAelbroeck, F. L. \& TASSI, E. 2012 A compressible Hamiltonian electromagnetic gyrofluid model. Commun. Nonlinear Sci. Numer. Simul. 17 (5), 2171-2178.

Waltz, R. E., Staebler, G. M., Dorland, W., Hammett, G. W., Kotschenreuther, M. \& Konings, J. A. 1997 A gyro-Landau-fluid transport model. Phys. Plasmas 4 (7), 2482-2496.

Webi, G. 2018 Magnetohydrodynamics and Fluid Dynamics: Action Principles and Conservation Laws, Lecture Notes in Physics, vol. 946. Springer.

Webb, G. M., Dasgupta, B., McKenzie, J. F., Hu, Q. \& Zank, G. P. 2014a Local and nonlocal advected invariants and helicities in magnetohydrodynamics and gas dynamics I: Lie dragging approach. J. Phys. A: Math. Gen. 47 (9), 095501.

Webb, G. M., Dasgupta, B., McKenzie, J. F., Hu, Q. \& Zank, G. P. $2014 b$ Local and nonlocal advected invariants and helicities in magnetohydrodynamics and gas dynamics: II. Noether's theorems and Casimirs. J. Phys. A: Math. Gen. 47 (9), 095502.

WeInberg, S. 2015 Lectures on Quantum Mechanics, 2nd edn. Cambridge University Press.

YoshidA, Z. \& MORRISON, P. J. 2014 A hierarchy of noncanonical Hamiltonian systems: circulation laws in an extended phase space. Fluid Dyn. Res. 46 (3), 031412.

YoshidA, Z. \& MORRISON, P. J. 2016 Hierarchical structure of noncanonical Hamiltonian systems. Phys. Scr. 91 (2), 024001.

Yoshida, Z., Morrison, P. J. \& Dobarro, F. 2014 Singular Casimir elements of the Euler equation and equilibrium points. J. Math. Fluid Mech. 16 (1), 41-57.

Yoshida, Z., Ohsaki, S., Ito, A. \& Mahajan, S. M. 2003 Stability of Beltrami flows. J. Math. Phys. 44 (5), 2168-2178. 